\title{
Proceedings of the 2018 ISNR Conference
}

\author{
Selected Abstracts of Conference Presentations at the 2018 International Society for Neurofeedback \\ and Research (ISNR) 26th Conference, Glendale, Arizona, USA
}

Citation: International Society for Neurofeedback and Research. (2018). Proceedings of the 2018 ISNR Conference. NeuroRegulation, 5(4), 150-174._http://dx.doi.org/10.15540/nr.5.4.150

Copyright: $\odot$ 2018. ISNR. This is an Open Access article distributed under the terms of the Creative Commons Attribution License (CC-BY).

\section{KEYNOTE PRESENTATIONS}

\section{Assessment and Outpatient Treatment of Addiction Using Neurofeedback and a Functional Medicine Approach \\ Susan K. Blank \\ Atlanta Healing Center, Atlanta, Georgia, USA}

The most common type of addiction treatment in the United States today is based on the Minnesota Model. This style of treatment relies on 12 Steps principles and was developed over 60 years ago. Despite a tremendous amount of research and new evidence-based practices, very few treatment centers incorporate innovations like neurofeedback.

In this session, we will look at and review several cases studies from the Atlanta Healing Center that illustrate important advances in treatment of the chronic brain disease of addiction. We will focus on the importance of making the proper diagnosis; medication-assisted recovery; evaluating cooccurring psychiatric, cognitive, and pain conditions; assessing the hormonal and nutritional status of patients; and providing treatment modalities like neurofeedback. Education of the family and patient is important and connecting with recovery support is essential for the patient to have the best possible outcome in the management of this potentially lifethreatening disease.

\section{The Effects and Mechanisms of Mindfulness Meditation, Cognitive Therapy, and Mindfulness-based Cognitive Therapy for Chronic Low Back Pain \\ Melissa Day \\ School of Psychology, University of Queensland, St. Lucia, Queensland, Australia}

Chronic low back pain (CLBP) is a pervasive, costly, and highly disabling condition. Research has shown that CLBP is inadequately managed solely by biomedical approaches alone. Thus, current guidelines put forth by the Centers for Disease Control and Prevention in the U.S. recommend nonpharmacological therapy as the first line approach to chronic pain management. In this context, there is evidence that Cognitive Therapy (CT) and mindfulness meditation (MM) programs are beneficial for a range of CLBP-related outcomes. Although not previously tested for CLBP management, evidence in other pain populations suggests that Mindfulnessbased Cognitive Therapy (MBCT) — which seamlessly integrates $\mathrm{CT}$ and MM techniques-might also be particularly well suited for improving pain, mood, and function.

An expanding body of research is investigating the potential neuromodulatory function of these psychosocial pain treatments. Although scarce research has examined brain state-related changes in the context of CT and MBCT for pain, within MM, several studies in pain samples have used electroencephalogram (EEG) at pre- and posttreatment to test the possible role of brain activity changes in association with improved pain-related outcomes. Results found MM was associated with power increases primarily in the alpha band, and this increase in alpha was suggested to play a key role in the effects of MM on pain. It is not known, however, if this potential neuromodulatory pathway is unique to $\mathrm{MM}$ as delivered as an isolated technique, or if it might also play a role in other similarly efficacious treatments, such as CT and MBCT.

In this session I will present data from a recently completed randomized controlled trial comparing $\mathrm{MM}$ versus $C T$ versus $M B C T$ within a CLBP sample. Treatment consisted of eight weekly, 2-hour groupdelivered sessions. EEG brain state data was obtained at pre- and posttreatment, as was selfreported pain-related outcome measures of pain interference, pain intensity, physical function, and depression. I will present (1) the treatment-related changes in the self-reported outcomes, (2) an 
analysis of change in brain activity across the three treatments, and (3) how potential changes in brain state are associated with changes in the self-reported outcomes.

\section{Is Addiction a Brain Disease? And Does It Matter? \\ Marc Lewis \\ University of Toronto, Toronto, Ontario, Canada}

Over the past 20 to 30 years, medical authorities have come to define and explain addiction as a brain disease. However, the domination of the disease model skews the science of addiction, diverts attention from key social-psychological factors, and results in potentially harmful trends in policy and clinical practice. In this talk I review the distortions and omissions of the classic brain disease model and point to problems in the treatment philosophy derived from it. I then outline an alternative model of addiction based on principles of learning and development. This model views addiction as an entrenched habit for regulating emotional needs, learned through the repeated pursuit of highly motivating but short-lived rewards. Developmental-learning models of addiction help explain individual differences in vulnerability (and recovery) based on early emotional difficulties and current psychological and social resources.

\section{INVITED PRESENTATIONS}

The Central Brain Mechanisms of Pain and the Neuromodulation Techniques for Addressing It Dirk DeRidder

Dunedin School of Medicine, University of Otago, Dunedin, New Zealand

Although chronic pain is one of the most important medical problems facing society, there has been limited progress in the development of novel therapies for this condition. The key to more successful pain treatment is to understand the mechanisms that generate and maintain chronic pain. Anatomically there exist at least two ascending pain input pathways and one descending pain inhibitory pathway. One input pathway encodes the painfulness, whereas the other pathway encodes the suffering or emotional pain associated with the painful stimulus. The pain inhibitory pathway probably encodes the percentage of the time the pain is dominantly present during the day. The anatomical pathways can be visualized using functional MRI meta-analyses, and LORETA EEG further shows that chronic pain is an imbalance between the ascending and descending pain inhibitory pathways. This is indeed confirmed both by activity, functional and effective connectivity EEG analyses.

Nonpharmacological treatment for chronic pain using spinal cord stimulation normalizes this imbalance, supporting the concept that pain is truly a balance disorder between pain input and pain suppression in the brain, and causally related to this imbalance. Pain thus is not merely the result of more pain input via the spinal cord or brainstem.

This imbalance mechanism, also known as thalamocortical dysrhythmia, might be universal in view of the pathophysiological analogy between pain, tinnitus, Parkinson's disease, and major depression. Furthermore, thalamocortical dysrhythmia and reward deficiency syndrome (obesity, addiction, $A D H D$, and personality disorders) may be two sides of the same coin as suggested by EEG source analyzed conjunction analyses between thalamocortical dysrhythmia and reward deficiency syndromes. As such, this new conceptualization of pain, Parkinson, tinnitus, depression, addiction, ADHD, OCD, and personality disorders as imbalances in the brain paves the way for neuromodulation techniques such as transcranial electrical stimulation and infraslow neurofeedback to normalize this imbalance.

\section{Integrating Mindfulness with Bio and Neurofeedback \\ Inna Khazan \\ Harvard Medical School, Boston, Massachusetts, USA}

Bio and neurofeedback are powerful treatment modalities shown to be effective at alleviating numerous psychophysiological conditions. Biofeedback provides a way to work with challenging conditions in cases for which other interventions have been unsuccessful, such as chronic pain, anxiety, headaches, and trauma. At the same time, bio/neurofeedback treatment itself can stall, leaving the client and the therapist feeling frustrated and unsure of how to proceed. These challenges include situations when the client is highly anxious about his or her physiological symptoms, feels pressure to "do things right," becomes easily overwhelmed with emotional stimuli, or is simply too distracted to attend to the computer screen for more than a few minutes at a time. Oftentimes, these challenges are due to the clients' unhelpful efforts to control the 
fundamentally uncontrollable aspects of their internal experience.

Mindfulness-based approach to bio/neurofeedback can help people experience change through mindful, nonjudgmental awareness and acceptance, providing the therapist and the client a way to work with what gets in the way of biofeedback success. In this talk participants will learn how to apply mindfulnessbased skills to their biofeedback practice in order to help their clients reap the benefits of biofeedback without getting stuck in unproductive attempts to control their internal experience.

\section{Update on the Work Towards CPT Codes and Third-Party Reimbursement \\ Mark Trullinger \\ NeuroThrive LLC, Parkville, Maryland, USA}

ISNR, in partnership with AAPB and BCIA, have taken significant strides in the past few years toward pushing for insurance reimbursement. This presentation will provide a macro-level progress report on the CPT coding workgroup trying to modernize our codes, petitions for inclusion as a recognized organization for AMA activities for CPT coding and Relative Value Unit (RVU) determinations, and national-level efforts for insurance reimbursement.

\section{STUDENT AWARD PRESENTATIONS - PLENARY SESSIONS}

\section{Tuning the Traumatized Brain: LORETA Z-score Neurofeedback and Heart Rate Variability Biofeedback for Chronic PTSD Ashlie Bell \\ Saybrook University, Oakland, California, USA \\ NeuroGrove, Lakewood, Colorado, USA}

Introduction. Neuroimaging studies have identified numerous abnormalities within the default mode (DMN), salience (SN), and central executive (CEN) neural networks of those suffering from PTSD (Lanius, Frewen, Tursich, Jetly, \& McKinnon, 2015). A systematic review of the literature revealed ten studies $(n=213)$ that examined neurofeedback as a method for altering these neural patterns and alleviating PTSD symptoms (Foster \& Thatcher, 2015; Gapen et al., 2016; Huang-Storms, Bodenhamer-Davis, Davis, \& Dunn, 2006; Kluetsch et al., 2014; Paret et al., 2014; Peniston \& Kulkosky, 1991; Peniston, Marrinan, Deming, \& Kulkosky, 1993; Pop-Jordanova \& Zorcec, 2004; Smith, 2008; van der
Kolk et al., 2016). These studies demonstrated mostly medium to large improvements following a variety of NF training modalities. Low-resolution electromagnetic tomography analysis (LORETA) zscore neurofeedback (LZNF) is a newer modality that is believed to produce more targeted and efficient outcomes than traditional modalities; however, it has not been adequately examined for the treatment of PTSD. This study is the first to examine the effectiveness of this modality using a controlled, experimental design.

Method. The purpose of this research study was to examine the effects of LZNF training, as compared to heart rate variability biofeedback (HRVB) training, on PTSD symptoms, autonomic regulation, and brainwave activation patterns in adults with chronic PTSD. Twenty-four participants were alternately assigned to receive 15 sessions of either LZNF ( $n=$ $12)$ or HRVB $(n=12)$ training. HRVB was chosen as an active control condition due to the ability to closely match many conditions to the LZNF group (i.e., time with trainer, resting state, body-computer interface, similar audio/visual feedback, etc.) while providing an ethical alternative for this sensitive population. Psychophysiological measurements (i.e., 19-channel EEG and HRV) were recorded before, during, and after a single session of training as well as before and after 15 training sessions. Psychosocial questionnaires were completed during the pre- and postintervention assessments.

Results. The data for this study is still being analyzed and thus results are not yet available; however, visual examination of the data and symptom reports suggest the results will be positive. The full results will be analyzed and ready to present before this ISNR conference. Paired and independent samples $t$-tests and Cohen's $d$ effect sizes are being utilized to examine both within- and between-group changes after 1 and 15 sessions. Pre-post changes will be analyzed for mean LORETA current source density (CSD) $z$-scores of three neural networks (i.e., DMN, $\mathrm{SN}$, and CEN); HRV metrics (i.e., standard deviation of $\mathrm{NN}$ intervals, root mean square of the successive difference, low-frequency power); and total scores on the PTSD Checklist for DMS-V and Beck Anxiety Inventory. I have hypothesized that LZNF will produce greater changes in LORETA CSD $z$-scores and PTSD symptoms, while HRVB will produce greater changes in HRV metrics.

Conclusion. This study is expected to provide important, preliminary data regarding the effectiveness of both LZNF and HRVB training on 
PTSD symptoms and HRV, as well as their differential effects on each of the neural networks suspected to underlie PTSD symptomology.

\section{References}

Foster, D. S., \& Thatcher, R. W. (2015). Surface and LORETA neurofeedback in the treatment of post-traumatic stress disorder and mild traumatic brain injury. In R. W. Thatcher \& D. S. Foster (Eds.), Z score neurofeedback: Clinical applications (pp. 59-92). San Diego, CA: Academic Press.

Gapen, M., van der Kolk, B. A., Hamlin, E., Hirshberg, L., Suvak, M., \& Spinazzola, J. (2016). A pilot study of neurofeedback for chronic PTSD. Applied Psychophysiology and Biofeedback, 41(3), 251-261. http://dx.doi.org/10.1007/s10484-015-9326-5

Huang-Storms, L., Bodenhamer-Davis, E., Davis, R., \& Dunn, J. (2006). QEEG-guided neurofeedback for children with histories of abuse and neglect: Neurodevelopmental rationale and pilot study. Journal of Neurotherapy, 10(4), 3-16. http://dx.doi.org/10.1300/J184v10n04_02

Kluetsch, R. C., Ros, T., Théberge, J., Frewen, P. A., Calhoun, V. D., Schmahl, C., ... Lanius, R. A. (2014). Plastic modulation of PTSD resting-state networks and subjective wellbeing by EEG neurofeedback. Acta Psychiatrica Scandinavica, 130(2), 123136. http://dx.doi.org/10.1111/acps.12229

Lanius, R. A., Frewen, P. A., Tursich, M., Jetly, R., \& McKinnon, M. C. (2015). Restoring large-scale brain networks in PTSD and related disorders: A proposal for neuroscientifically-informed treatment interventions. European Journal of Psychotraumatology, $\quad$ 6(1). $\quad$ http://dx.doi.org/10.3402 lejpt.v6.27313

Paret, C., Kluetsch, R., Ruf, M., Demirakca, T., Hoesterey, S., Ende, G., \& Schmahl, C. (2014). Down-regulation of amygdala activation with real-time fMRI neurofeedback in a healthy female sample. Frontiers in Behavioral Neuroscience, 8, 299. http://dx.doi.org/10.3389/fnbeh.2014.00299

Peniston, E. G., \& Kulkosky, P. J. (1991). Alpha-theta brainwave neurofeedback therapy for Vietnam veterans with combatrelated post-traumatic stress disorder. Medical Psychotherapy, 4, 47-60.

Peniston, E. G., Marrinan, D. A., Deming, W. A., \& Kulkosky, P. J. (1993). EEG alpha-theta brainwave synchronization in Vietnam theater veterans with combat-related post-traumatic stress disorder and alcohol abuse. Advances in Medical Psychotherapy, 6, 37-50.

Pop-Jordanova, N., \& Zorcec, T. (2004). Child trauma, attachment and biofeedback mitigation. Prilozi / Makedonska Akademija Na Naukite I Umetnostite, Oddelenie Za Biološki I Medicinski Nauki = Contributions / Macedonian Academy of Sciences and Arts, Section of Biological and Medical Sciences, 25(1-2), 103-114.

Ros, T., Baars, B. J., Lanius, R. A., \& Vuilleumier, P. (2014). Tuning pathological brain oscillations with neurofeedback: A systems neuroscience framework. Frontiers in Human Neuroscience, 8 , 1008. http://dx.doi.org/10.3389/fnhum.2014.01008

Smith, W. D. (2008). The effect of neurofeedback training on PTSD symptoms of depression and attention problems among military veterans. Capella University. Retrieved from http://gradworks.umi.com/33/15/3315214.html

van der Kolk, B. A., Hodgdon, H., Gapen, M., Musicaro, R., Suvak, M. K., Hamlin, E., \& Spinazzola, J. (2016). A randomized controlled study of neurofeedback for chronic PTSD. PLoS ONE, 11(12), e0166752. http://dx.doi.org /10.1371/journal.pone.0166752

\author{
Personalized EEG-Neurofeedback as a \\ Treatment for ADHD \\ Helene Brisebois ${ }^{1}$, Caroline Dupont ${ }^{2}$ \\ ${ }^{1}$ Collège Montmorency, Laval, Quebec, Canada \\ ${ }^{2}$ University of Montreal, Montreal, Quebec, Canada
}

Several neurophysiological subtypes based on electroencephalographic (EEG) biomarkers have been identified in attention-deficit/hyperactivity disorder (ADHD; Johnstone, Gunkelman, \& Lunt, 2005). However, most studies investigating the efficacy of neurofeedback (NFB) as a treatment for $A D H D$ use uniform treatment protocols that are not taking into account individual EEG biomarkers (Arns, de Ridder, Strehl, Breteler, \& Coenen, 2009). A recent pilot study suggests that personalizing NFB protocols to individual EEG biomarkers of ADHD might lead to increased specificity and efficacy of treatment (Arns, Drinkenburg, \& Kenemans, 2012). Hence, the objective of this presentation is to investigate the effects of personalized EEG-NFB as a treatment for $A D H D$. It will provide an overview of personalized EEG-NFB protocols for ADHD and introduce results from a pilot project that aimed to integrate a neurofeedback clinic as part of the services offering of the Office for Students with Disabilities in a Canadian college. A hundred and eight college students with a diagnosis of ADHD received free personalized EEG-NFB twice a week over a period of 4 months. Half of the participants was randomly assigned to the experimental condition. The other half was put on a waiting list to serve as a control group and received treatment later. Restingstate EEG signals were recorded to evaluate overall brain activity pre- and posttraining, and to determine individual EEG-biomarkers for selection of personalized treatment protocol. ADHD behavioral symptoms were assessed pre- and posttraining using the Conners' Adult ADHD Rating Scale (CAARS-S:L), the Integrated Visual and Auditory Continuous Performance Test (IVA-2) and assessment of executive functions. A significant change was observed in subjects trained in EEG-NFB, both in brain activation patterns and at the behavioral level. More specifically, normalization of targeted resting brain waves was observed in the experimental group. Results from this pilot project demonstrate the feasibility of personalizing NFB protocols to individual EEG biomarkers of ADHD and the efficacy of NFB as a treatment for ADHD. On a broader level, this presentation will allow for a better understanding of the impact of neurofeedback training on neural and behavioral correlates of ADHD. 


\section{References}

Arns, M., de Ridder, S., Strehl, U., Breteler, M., \& Coenen, A. (2009). Efficacy of neurofeedback treatment in ADHD: The effects on inattention, impulsivity and hyperactivity: A metaanalysis. Clinical EEG Neuroscience, 40(3), 180-189. http://dx.doi.org/10.1177/155005940904000311

Arns, M., Drinkenburg, W., \& Kenemans, J. L. (2012). The effects of qEEG-informed neurofeedback in ADHD: An open-label pilot study. Applied Psychophysiology and Biofeedback, 37(3), 171-180. http://dx.doi.org/10.1007/s10484-012-9191-4

Johnstone, J., Gunkelman, J., \& Lunt, J. (2005). Clinical database development: Characterization of EEG phenotypes. Clinical EEG and Neuroscience, 36(2), 99-107. http://dx.doi.org $/ 10.1177 / 155005940503600209$

\section{The Nonlinear Brain: Investigating Neural Entrainment Using Missing Pulse Rhythms Charles Wasserman, Yi Wei, Erika Skoe, Heather Read, Edward Large \\ University of Connecticut, Mansfield, Connecticut, USA}

Introduction. Many rhythm perception experiments employ simple isochronous rhythms, in which synchronous neural or behavioral responses are observed (Bauer, Kreutz, \& Herrmann, 2015; Repp, 2005a, 2005b). However, responses at the stimulus frequency do not allow one to distinguish whether synchrony occurs as a response to a common input or as the result of an emergent population oscillation that entrains at a particular frequency. It is possible to create a rhythm with no spectral energy at the pulse frequency by manipulating the number of events that occur anti-phase $\left(180^{\circ}\right)$ versus in-phase $\left(0^{\circ}\right)$ with the basic rhythmic cycle. Dynamical analysis predicts neural oscillation will emerge at such a "missing" pulse frequency (Large, 2010). Previous studies have shown that subjects tap along to complex rhythms at the missing pulse frequency (Large, Herrera, \& Velasco, 2015)-a finding that supports the prediction, and responses at missing pulse frequencies have been seen in auditory brain areas using magnetoencephalography (MEG; Tal et al., 2017).

Aims. This study aimed to investigate whether the sensorimotor system, as measured by 32-channel cortical EEG, would entrain to a complex rhythm at the pulse frequency even when the complex rhythm contained no spectral power at that frequency.

Methods. The experiment utilized four different rhythms of varying complexity (one simple, two complex, and one random rhythm) created from 100 ms tones with a $200 \mathrm{~Hz}$ fundamental frequency (F0). Offline the EEG was decomposed into the corticalsteady state response (SS-EP) and the subcortical frequency following response (FFR). Fast Fourier Transform (FFT) of the Hilbert envelope showed energy at the repetition frequency $(2 \mathrm{~Hz})$ for the simple rhythm, but no spectral energy at the missing pulse frequency $(2 \mathrm{~Hz})$ for the complex rhythms. EEG responses to these stimuli were examined for evidence of neural oscillations and power modulations at the missing pulse frequency predicted by dynamical analysis.

Results. We report evidence of responses in the EEG to the pulse frequency of missing pulse rhythms. We also note a differing topography of power at the pulse frequency across the scalp for the complex rhythms versus the simple and random rhythms.

Conclusions. These data support the theory that rhythmic synchrony occurs as the result of an emergent population oscillation that entrains at this particular frequency. Additional analyses examined whether the FFR to the F0 is modulated by whether the stimuli are perceived as being on-beats or offbeats in the rhythmic context.

\section{References}

Bauer, A.-K. R., Kreutz, G., \& Herrmann, C. S. (2015). Individual musical tempo preference correlates with EEG beta rhythm. Psychophysiology, 52(4), 600-604. http://dx.doi.org/10.1111 /psyp. 12375

Large, E. W. (2010). Neurodynamics of music. In M. R. Jones, R. R. Fay, \& A. N. Popper (Eds.), Springer Handbook of Auditory Research, Volume 36: Music perception (Vol. 36, pp. 201231). Springer Science+Business Media, LLC. Retrieved from http://link.springer.com/10.1007/978-1-4419-6114-3 7

Large, E. W., Herrera, J. A., \& Velasco, M. J. (2015). Neural networks for beat perception in musical rhythm. Frontiers in Systems Neuroscience, 9, 159. http://dx.doi.org/10.3389 /fnsys.2015.00159

Repp, B. H. (2005a). Rate limits of on-beat and off-beat tapping with simple auditory rhythms: 2 . The roles of different kinds of accent. Music Perception, 23(2), 165-188.

Repp, B. H. (2005b). Sensorimotor synchronization: A review of the tapping literature. Psychonomic Bulletin \& Review, 12(6), 969992. http://dx.doi.org/10.3758/BF03206433

Tal, I., Large, E. W., Rabinovitch, E., Wei, Y., Schroeder, C. E., Poeppel, D., \& Golumbic, E. Z. (2017). Neural entrainment to the beat: The "missing-pulse" phenomenon. The Journal of Neuroscience, 37(26), 6331-6341. http://dx.doi.org /10.1523/JNEUROSCI.2500-16.2017 


\section{STUDENT AWARD PRESENTATIONS - POSTERS}

\author{
Noninvasive Cranial Nerve Stimulation for \\ Human Cognitive Performance Enhancement \\ Taylor Hearn, Sarah Wyckoff, Stephen Helms Tillery, \\ William Tyler \\ Arizona State University, Tempe, Arizona, USA
}

Electrical stimulation of various cranial and spinal nerves is a rapidly growing area of study. Noninvasive approaches especially provide a safer, less expensive alternative to the pharmacological treatment of various psychological conditions. Specifically, vagus nerve stimulation (VNS) has been shown alleviate symptoms of major depressive disorder, trigeminal nerve stimulation (TNS), epilepsy, and cervical spinal nerve stimulation (CNS), stress (Berry et al., 2013; DeGiorgio et al., 2013; Tyler et al., 2015). These stimulation sites are all believed to innervate the locus coeruleus-norepinephrine system (Berry et al., 2013). Taking into account the extensive role norepinephrine plays in various executive functions, these stimulation techniques should be able to affect executive functioning in healthy subjects as well (Sara, 2009). As such, our protocol seeks to elucidate the neuromodulatory effects of noninvasive cranial nerve stimulation on attention.

A passive auditory oddball task was selected to measure attention. Subjects were instructed to listen to a series of $100 \mathrm{~ms}$ tones followed by $500 \mathrm{~ms}$ of silence. For each $600 \mathrm{~ms}$ trial, there was an $82 \%$ chance the tone would be at $750 \mathrm{~Hz}$ and an $18 \%$ chance it would be at $1,500 \mathrm{~Hz}$. Tones were presented until 150 tones at $1,500 \mathrm{~Hz}$ were presented. Subjects were not required to physically or consciously respond to either type of tone. This task was created entirely with custom MATLAB (Natick, MA) software.

CNS, TNS, and VNS were all delivered using a custom, current-controlled stimulator connected to $2.5 \mathrm{~cm}$ round Axelgaard PALS electrodes (Fallbrook, CA). Stimulation trains were delivered for $10 \mathrm{~min}$ between oddball tasks. Each train was symmetrically biphasic, charge-balanced, and cathodic-first. Subjects were randomly assigned to receive either CNS, TNS, or VNS at 30,300 , or $3,000 \mathrm{~Hz}$ with pulse durations of 50,350 , and $50 \mu \mathrm{s}$, respectively. After all testing, subjects completed a subjective report stimulation to describe the stimulation experience.
Electroencephalography (EEG), electrocardiography, galvanic skin response, respiratory rate, and hand temperature were all utilized to assess the physiological responses to stimulation.

Data collection is ongoing. EEG data will be averaged across subjects for each stimulation location and parameter set and presented as voltage traces and spectrograms to examine effects in both time and frequency domains. Physiological data will be averaged similarly to EEG data. Subjects' subjective experiences will also be reported.

\section{References}

Berry, S. M., Broglio, K., Bunker, M., Jayewardene, A., Olin, B., \& Rush, A. J. (2013). A patient-level meta-analysis of studies evaluating vagus nerve stimulation therapy for treatment-resistant depression. Medical Devices: Evidence and Research, 6, 17-35. http://dx.doi.org/10.2147 IMDER.S41017

DeGiorgio, C. M., Soss, J., Cook, I. A., Markovic, D., Gornbein, J., Murray, D., ... Heck, C. N. (2013). Randomized controlled trial of trigeminal nerve stimulation for drug-resistant epilepsy. Neurology, $\quad 80(9), \quad 786-791 . \quad$ http://dx.doi.org/10.1212 /WNL.0b013e318285c11a

Sara, S. J. (2009). The locus coeruleus and noradrenergic modulation of cognition. Nature Reviews Neuroscience, 10(3), 211-223. http://dx.doi.org/10.1038/nrn2573

Tyler, W. J., Boasso, A. M., Mortimore, H. M., Silva, R. S., Charlesworth, J. D., Marlin, M. A., ... Pal, S. K. (2015) Transdermal neuromodulation of noradrenergic activity suppresses psychophysiological and biochemical stress responses in humans. Scientific Reports, 5, 13865. http://dx.doi.org/10.1038/srep13865

The Effects of ALAY and High Beta Down-train Neurofeedback for Patients Who Comorbid with Major Depressive Disorder and Anxiety Symptoms

San Yu Wang ${ }^{1}$, I-Mei Lin ${ }^{1}$, Yu-Che Tsai', Cheng-Fang Yen $^{1}$, Yi-Chun Yeh ${ }^{1}$, Mei-Feng Huang ${ }^{1}$, Tai Ling Liu', Peng-Wei Wang', Huang Chi Lin', Yu Lee ${ }^{2}$, Nien-Mu $\mathrm{Chiu}^{2}$, Chi-Fa Hung ${ }^{2}$

${ }^{1}$ Kaohsiung Medical University, Kaohsiung City, Taiwan, Taiwan

${ }^{2}$ Kaohsiung Chang Gung Memorial Hospital, Kaohsiung City,

Taiwan, Taiwan

Background and Description. Previous studies indicated that Frontal Alpha Asymmetry (FAA) and parietal hyperactivity among patients who comorbid with Major Depressive Disorder (MDD; Bruder et al., 1997; Mathersul, Williams, Hopkinson, \& Kemp, 2008 ) and high-level anxiety symptoms. The purpose of this study was to examine the effects of alpha asymmetry (ALAY) and high beta down-train (BETA) neurofeedback protocols on emotional symptoms and electroencephalogram (EEG) among patients with MDD and anxiety symptoms. 
Methods. Patients with MDD were referred by psychiatrists based on DSM- 5 criteria, and with the scores of Beck Depression Inventory II (BDI-II) and Beck Anxiety Inventory (BAI) which were higher than 14 and 8. Eight-seven participants were assigned to ALAY neurofeedback (ALAY group; $n=24$ ), high beta down-train neurofeedback (BETA group), and the control group $(n=23)$. All participants received BDI$\mathrm{II}, \mathrm{BAI}$, and a 5-min resting EEG with eye-closed measurement by using a 19-channel EEG cap with BrainAvatar equipment (BrainMaster Technologies, Inc., Bedford, Ohio) for pretest and posttest. The EEG raw signals were analyzed to alpha power (8$12 \mathrm{~Hz})$ and high beta power $(20-32 \mathrm{~Hz})$, and then calculated to the A1 score (log [F4 alpha] - log [F3 alpha]) and high beta at P3 and P4. Both neurofeedback groups received 60 -min treatment, twice a week, for 10 consecutive sessions by using ProComp Infiniti (Thought Technology Ltd., Montreal, Quebec, Canada). The goal of the ALAY group was to increase the A1 score, while the BETA group was to decrease high beta at P3 and P4.

Results. There was a significant decrease in the symptoms of depression and anxiety in both the ALAY group: $F(1,23)=26.07, p<.001 ; F(1,23)=$ $13.73, p=.001$; and the BETA group: $F(1,22)=24.27$, $p<.001 ; F(1,22)=33.06, p<.001$. Lower anxiety level was also found in the posttest in ALAY and BETA groups compared to the control group, $F(2,67)$ $=9.48, p<.001$. However, lower level of depressive symptoms at posttest was found only in the BETA group compared to the control group, $F(2,67)=4.56$, $p=.014$. There was a significant decrease in P3 high beta in the BETA group at posttest than that at pretest, $F(1,22)=8.64, p=.008$; while significant increase in P3 high beta in the control group at posttest than that at pretest, $F(1,22)=6.28, p=.020$. However, there was no significant interaction effect which was found in A1 score, F3 total alpha, or F4 total alpha between the ALAY group and the control group, $F(1,44)=0.91, p=.345 ; F(1,45)=0.002, p=$ $.967 ; F(1,44)=0.02, p=.882$.

Conclusion. This study indicated that both ALAY and BETA neurofeedback protocols significantly decreased the symptoms of depression and anxiety among the patients who comorbid with MDD and high-level anxiety. Moreover, there was a significant decrease in high beta activity at posterior region (P3) which was found in high beta down-train neurofeedback protocol.

\section{References}

Bruder, G. E., Fong, R., Tenke, C. E., Leite, P., Towey, J. P., Stewart, J. E., ... Quitkin, F. M. (1997). Regional brain asymmetries in major depression with or without an anxiety disorder: A quantitative electroencephalographic study. Biological Psychiatry, 41(9), 939-948. http://dx.doi.org /10.1016/S0006-3223(96)00260-0

Grin-Yatsenko, V. A., Baas, I., Ponomarev, V. A., \& Kropotov, J. D. (2009). EEG power spectra at early stages of depressive disorders. Journal of Clinical Neurophysiology, 26(6), 401406. http://dx.doi.org/10.1097/WNP.0b013e3181c298fe

Mathersul, D., Williams, L. M., Hopkinson, P. J., \& Kemp, A. H. (2008). Investigating models of affect: Relationships among EEG alpha asymmetry, depression, and anxiety. Emotion, 8(4), 560-572. http://dx.doi.org/10.1037/a0012811

Yamada, M., Kimura, M., Mori, T., \& Endo, S. (1995). EEG power and coherence in presenile and senile depression. Characteristic findings related to differences between anxiety type and retardation type. Nihon Ika Daigaku Zasshi = Journal of Nippon Medical School, 62(2), 176-185. http://dx.doi.org /10.1272/jnms1923.62.176

Lateralized Readiness Potentials in Children with Autism Spectrum Disorder During Posner Cueing Task: An Event-related EEG Study

Guela Sokhadze', Estate Sokhadze ${ }^{2}$, Manuel Casanova ${ }^{2}$

${ }^{1}$ University of Louisville, Louisville, Kentucky, USA

${ }^{2}$ University of South Carolina, School of Medicine-Greenville, Greenville, South Carolina, USA

Background. Autism spectrum disorder (ASD) is a developmental disorder characterized by social communication deficits, and engagement in restricted, stereotyped behaviors. An estimated $80 \%$ of individuals with ASD also display dyspraxia (Weimer, Schatz, Lincoln, Ballantyne, \& Trauner, 2001; Dowell, Mahone, \& Mostofsky, 2009), a condition involving difficulties in motor coordination and sequencing, as well as speech production. However, it is unclear how the processing, preparation, and execution phases of motor movement are affected by dyspraxia in ASD. We examined EEG activity and behavioral indices in children diagnosed with ASD during performance of a visuo-motor spatial attention task.

Methods. Participants included 30 children diagnosed with ASD (15.6 \pm 3.8 years old, 8 girls), and an age-matched control group of 30 typicallydeveloping children (TD; $15.7 \pm 3.9$ years old, 7 girls). Subjects performed a modified Posner's attentional cueing task (Posner, 1980). In each trial, subjects were initially presented a visual "cue" stimulus on left or right side of the screen. After a 1- s delay, a "target" stimulus appeared on the same (congruent, $80 \%$ ) or opposite (incongruent, $20 \%$ ) side from the cue, and subjects used a left- or right-handed button press to 
indicate the position of the target. In half of the trials, a more complex diagonal stimulus presentation was used. EEG data was collected for analysis of several event-related potentials (ERPs), including the lateralized readiness potential (LRP), a measurement of asymmetric brain activity that reflects preparation of contralateral limb movement (Eimer, 1998).

Results. Reaction time (RT) was lower for congruent trials compared to incongruent trials for both ASD (401 vs. $481 \mathrm{~ms}, p<.0001)$ and TD (339 vs. $374 \mathrm{~ms}$, $p<.001)$. Across all four task conditions, ASD group exhibited longer RTs and higher error rates compared to TD (441 vs. $358 \mathrm{~ms}, p<.001 ; 7.4$ vs. 0.8 errors, $p$ $<.0001)$. Furthermore, increased task complexity resulted in lengthened RT in ASD group (447 vs. 430 $\mathrm{ms}, p<.05$ ), and in TD group (376 vs. $360 \mathrm{~ms}, p<$ .01). While the amplitude the early (pretarget) component of LRP was significantly higher in ASD compared to TD $(-0.95$ vs. $-0.23, p<.05)$, the late component (posttarget) showed no group differences ( -0.53 vs. -0.50 , n.s.). Moreover, analysis of ERPs showed several differences between ASD and TD groups, including frontal N100 amplitude, N100 latency, and N200 amplitude.

Discussion and Conclusions. Shorter RTs to congruent trials in ASD and TD suggest that both groups exhibited an attentional bias toward the cued side. However, ASD group had higher RTs and lower accuracy regardless of trial condition, indicating poorer performance compared to TD. EEG analysis demonstrated that ASD group exhibited differences in the early ERPs and LRP, indicating differences at the early cognitive phase of stimulus processing and movement preparation rather that at the late motor execution phase. A more in-depth understanding of abnormalities in LRP and other ERPs during motor task performance could shed light on the underlying neuropathology of dyspraxia in autism and could potentially serve as a useful biomarker for early diagnosis.

\section{References}

Dowell, L. R., Mahone, E. M., \& Mostofsky, S. H. (2009). Association of postural knowledge and basic motor skill with dyspraxia in autism: Implication for abnormalities in disturbed connectivity and motor learning. Neuropsychology, 23(5), 563570. http://dx.doi.org/10.1037/a0015640

Eimer, M. (1998). The lateralized readiness potential as an on-line measure of central response activation processes. Behavior Research Methods, Instruments, \& Computers, 30(1), 146156. http://dx.doi.org/10.3758/BF03209424

Posner, M. I. (1980). Orienting of attention. Quarterly Journal of Experimental Psychology, 32(1), 3-25. http://dx.doi.org $/ 10.1080 / 00335558008248231$
Weimer, A. K., Schatz, A. M., Lincoln, A., Ballantyne, A. O., \& Trauner, D. A. (2001). "Motor" impairment in Asperger syndrome: Evidence for a deficit in propioception. Journal of Developmental \& Behavioral Pediatrics, 22(2), 92-101. http://dx.doi.org/10.1097/00004703-200104000-00002

\section{PLENARY SESSION PRESENTATIONS}

\section{The Importance of Morphology and Montaging in EEG \\ Tiffany Thompson \\ NeuroField Neurotherapy, Santa Barbara, California, USA}

Reading the raw EEG is an artform that is essential knowledge-base of any practitioner using EEG to assess and diagnose their patients' conditions. Spindles, triangular shapes, sinusoidal, monomorphic, and archiform waveforms are just a few telling morphological signs that are imperative in understanding what is really going on. Does the waveform wax and wane? Does it travel in spindles or bursts? Does it appear only a few times in the record? What if it is rhythmic? These temporal dynamics are also imperative in a proper assessment of the person. When looking at the raw waveform, you will learn more than what any qEEG, alone, can tell you.

Through exploring the more insidious forms of artifact (i.e., electricity, channel noise, mixed metals, etc.) to detecting less commonly seen morphological forms in the EEG (i.e., lambda, mu, OIRDA, beta spindles, etc.), this lecture will guide the clinician through some of the more advanced ways of interpreting EEG so that the qEEG does not mislead one into misdiagnosis.

We are privileged to have many analysis and diagnostic tools to help us dissect, spatially and temporally analyze, condense, and summate the EEG into neat and tidy diagrams, but we fail our patients and our profession if we miss the devils in the details.

Finally, montages are necessary to understand the many ways in which we can assess and view the EEG. There is no best montage for all purposes. While linked ears can provide a global view, it is prone to contamination if there is a strong temporal finding or if there is contamination otherwise in the ear electrodes. Average and weighted average montages (such as the Laplacian and Hjorth montages), will highlight any local phenomena, and will uncover any significant temporal component, but will fail us to see global information. Bipolar montages are excellent for displaying phase 
reversals, which are indispensable in issues of head injury and seizure focus.

\section{References}

Buzsáki, G. (2006). Rhythms of the brain. New York, NY: Oxford University Press.

Buzsáki, G., \& Draguhn, A. (2004). Neuronal oscillations in cortical networks. Science, 304(5679), 1926-1929. http://dx.doi.org/10.1126/science.1099745

Sporns, O., Tononi, G., \& Edelman, G. M. (2000a). Connectivity and complexity: The relationship between neuroanatomy and brain dynamics. Neural Networks, 13(8-9), 909-922. http://dx.doi.org/10.1016/S0893-6080(00)00053-8

Sporns, O., Tononi, G., \& Edelman, G. M. (2000b). Theoretical neuroanatomy: Relating anatomical and functional connectivity in graphs and cortical connection matrices. Cerebral Cortex, 10(2), 127-141. http://dx.doi.org/10.1093/cercor/10.2.127

Stern, J. M. (2005). Atlas of EEG patterns (2nd ed.). Philadelphia, PA: Lippincott Williams \& Wilkins.

The Impact of Using Effective Connectivity Measures (Granger Causality) in Guiding Neurofeedlback

Robert Coben, Anne Stevens

Integrated Neuroscience Services, Fayetteville, Arkansas, USA

Over the past several years, we have seen advancements in the ways we assess coherence and connectivity that provide great insights into brain functioning (Coben, Mohammad-Rezazadeh, \& Cannon, 2014). This understanding has led to approaching coherence in a multivariate fashion that enhances its accuracy (Kuś, Kamińshi, \& Blinowska, 2004). Multivariate autoregressive statistical tools have become critical to this endeavor. Such techniques enable us to measure effective connectivity in a source localized fashion such that we can image reciprocal causality and influence. This accuracy in depicting neural networks gets us closer to the real signals in the brain. This led to an enhancement in how we do neurofeedback training which now uses four sensors and trains coherence in a multivariate fashion (Coben, Middlebrooks, Lightstone, \& Corbell, 2018).

We have adopted a theory that states the more accurate our assessment of connectivity and the source of the activity then the more effective our attempts at neurofeedback may be. In this pilot study we evaluated the effects of basing our neurofeedback (multivariate coherence training) protocols on coherence measures as compared to multivariate autoregressive effective connectivity that uses sources to estimate reciprocal causality (see Friston, Moran, \& Seth, 2013). We sampled 45 subjects with various presenting complaints and divided up into three groups. All subjects had their neurofeedback protocols developed based on qEEG methods. Group 1 was based on ICA and effective connectivity Granger causality, and groups 2 and 3 were based on correlational coherence measures. We used two separate comparison groups, one served as a withingroup and the other a between-group comparison.

All subjects underwent between 12 and 15 neurofeedback sessions followed by another assessment period. Dependent measures included EEG comparisons of power and graph theory metrics of effective connectivity. Clinical comparisons were also made based on rating of progress to measure their symptoms change over this period of time. Preliminary data analysis has shown that all groups show changes and gains from their neurofeedback training, but that the group that had their protocols based on effective connectivity measures showed greater clinical and EEG changes. The implications of these findings will be presented.

\section{References}

Coben, R., Middlebrooks, M., Lightstone, H. \& Corbell, M. (in press). Four- channel multivariate coherence training: Development and evidence in support of a new form of neurofeedback. Frontiers in Neural Technology.

Coben, R., Mohammad-Rezazadeh, I., \& Cannon, R. L. (2014). Using quantitative and analytic EEG methods in the understanding of connectivity in autism spectrum disorders: A theory of mixed over-and under-connectivity. Frontiers in Human Neuroscience, 8, 45. http://dx.doi.org/10.3389 Ifnhum.2014.00045

Friston, K., Moran, R., \& Seth, A. K. (2013). Analysing connectivity with Granger causality and dynamic causal modelling. Current Opinion in Neurobiology, 23(2), 172-178. http://dx.doi.org/10.1016/j.conb.2012.11.010

Kuś, R., Kamiński, M., \& Blinowska, K. J. (2004). Determination of EEG activity propagation: Pair-wise versus multichannel estimate. IEEE Transactions on Biomedical Engineering, 51(9), 1501-1510. http://dx.doi.org/10.1109 /TBME.2004.827929

\section{Trends in Scientific Research Reflect and Predict the Clinical Relevance of (EEG) Biomarkers \\ Andrè Keizer \\ qEEG-Pro, Eindhoven, Brabant, Netherlands}

QEEG-guided neurofeedback is based on interpreting abnormalities in the resting-state EEG in relationship with psychopathology. Setting up effective neurofeedback treatment protocols relies on the correct interpretation of individual qEEG profiles in relationship with the symptoms of the patient. Scientific studies have demonstrated associations between certain deviations in resting-state EEG and 
specific psychological disorders. The most wellknown link is that of excess theta/beta ratio in relation with ADHD (e.g., Arns, Conners, \& Kraemer, 2013).

Recent approval of the FDA for an ADHD diagnostic test based on excess theta/beta ratio illustrates that this "EEG biomarker" is both meaningful and reliable. Other markers for psychopathology include "alpha asymmetry" for depression (Thibodeau, Jorgensen, \& Kim, 2006) and excess beta power for anxiety and insomnia (Pavlenko, Chernyi, \& Goubkina, 2009; Perlis, Merica, Smith \& Giles, 2001). However, a modern qEEG report will contain many different and detailed analyses of an individual EEG. Moreover, the number of analyses that can be performed on EEG data has been increasing rapidly. Interpreting the clinical relevance of these analyses for the treatment of an individual patient depends on the scientific studies demonstrating links between these measures and the symptoms of the patient.

In the current presentation, the trends in qEEG research will be discussed and an attempt will be made to assess the relevance of different qEEG analyses for clinical purposes using a systematic analysis of the scientific literature. The main approach is to analyze the number of papers published on a particular search term (reflecting a certain EEG biomarker) per year. A secondary approach is to analyze the number of citations per year to seminal papers which describe a new EEG biomarker. Finally, comparisons with scientific literature in different fields may provide useful analogies. For example, the interpretation of an individual blood test for cancer relies on scientific research on the association between certain biomarkers (e.g., certain proteins) and the presence of a tumor. How did scientific research on these "tumor markers" evolve and eventually lead up to the use of tumor markers in clinical testing today, and what can this tell us about the current state-of-the-art and future clinical relevance of different EEG analyses?

One hypothesis is that papers which demonstrate a promising new biomarker will show a continuous increase in citations per year when the biomarker can be replicated and when it is useful as a diagnostic tool in clinical practice. In contrast, when such a study cannot be replicated it may show an initial increase of citations, but the number of citations per year will inevitably go down after this initial increase. In the latter case, it can be concluded that the biomarker is not useful in clinical practice. Analyzing trends in the scientific literature to predict clinical relevance of potential EEG biomarkers is novel and relevant approach which may have important implications for the scientific and clinical field of qEEG and neurofeedback.

\section{References}

Arns, M., Conners, C. K., \& Kraemer, H. C. (2013). A decade of EEG theta/beta research in ADHD: A meta-analysis. Journal of Attentional Disorders, 17(5), 374-383. http://dx.doi.org $/ 10.1177 / 1087054712460087$

Pavlenko, V. B., Chernyi, S. V., \& Goubkina, D. G. (2009). EEG correlates of anxiety and emotional stability in adult healthy subjects. Neurophysiology, 41(5), 337-345. http://dx.doi.org /10.1007/s11062-010-9111-2

Perlis, M. L., Merica, H., Smith, M. T., \& Giles, D. E. (2001). Beta EEG activity and insomnia. Sleep Medicine Reviews, 5(5), 363-374. http://dx.doi.org/10.1053/smrv.2001.0151

Thibodeau, R., Jorgensen, R. S., \& Kim, S. (2006). Depression, anxiety, and resting frontal EEG asymmetry: A meta-analytic review. Journal of Abnormal Psychology, 115(4), 715-729. http://dx.doi.org/10.1037/0021-843X.115.4.715

\section{Understanding the Mysterious $40 \mathrm{~Hz}$ Brain System for Attention, Learning, and Feeling Good \\ Jonathan Cowan ${ }^{1}$, Estate Sokhadaze ${ }^{2}$ \\ ${ }^{1}$ NeuroTek, LLC, Goshen, Kentucky, USA \\ 2University of South Carolina, School of Medicine Greenville, Greenville, South Carolina, USA}

Evidence from a wide variety of studies and authors supports a new synthesis of understanding regarding the $40 \mathrm{~Hz}$ brain scanning system and its role in attention, understanding, learning, and creating positive feelings as a reward for the effort. It is a basic foundation for the individual's survival, promoting their effective responses to new discoveries and situations. We have therefore named this brain system Neureka! (short for Neural Eureka!). This understanding builds upon Llinas' discovery of the "Event Binding Rhythm" (Llinas, Ribary, Contreras, \& Pedroarena,1998), which scans the cortical layers from front to back 40 times a second and reports back to its origin, the centrally located nuclei in the thalamus, all of which we will review here.

These nuclei synthesize all this information and send out modified $40 \mathrm{~Hz}$ scanning rhythms which look for additional information to add to the understanding of a particular new event. This looping information exchange continues until the event is evaluated and a response is created. Salient events are stored in short-term memory, particularly in the prefrontal cortex (PFC). Short-term memory is then converted to longer term memory, particularly if dopamine, norepinephrine, and/or other neuromodulators are released. There is evidence that both of these 
neuromodulators are released in the PFC, particularly near FPz. Dopamine also creates a variety of positive feelings when it is released there.

We will review several lines of evidence about the Neureka! brain system from fMRI and other scans (Knutson, Fong, Bennett, Adams, \& Hommer, 2003), and complement them with our EEG studies based on the Neureka! measurement, which selectively clarifies this particular $40 \mathrm{~Hz}$ rhythm and separates it from the other $40 \mathrm{~Hz}$ activity passing through more peripheral parts of the thalamus.

These studies show that neurofeedback training of Neureka! enhances memory and happiness (for at least four months) and decreases depressed feelings. It also improves memory and attention measurements (Sokhadze \& Daniels, 2016). Previous studies (Cowan \& Albers, 2011; Cowan \& Starman, 2017; Rubik, 2011) demonstrate clear relationships between increases in Neureka! amplitude and love, happiness, satisfaction, gratitude, and appreciation. We will review studies where neurofeedback training using the Neureka! measure (Sokhadze, 2012) was used to improve behavioral symptoms in children with autism (Wang et al., 2016). Furthermore, the $40 \mathrm{~Hz}$ rhythm was used to distinguish the emotional reaction to drug and stress cues of substance abusers from those with comorbid PTSD (Sokhadze et al., 2009).

\section{References}

Cowan, J., \& Albers, S. (2017). Manual for the Peak Brain Happiness Trainer. Goshen, KY: Peak Achievement Training.

Cowan, J. D., \& Starman, J. D. (2011). Understanding and activating your brain's pleasure systems. Retrieved from http://www.peakachievement.com/UABC.pdf

Knutson, B., Fong, G. W., Bennett, S. M., Adams, C. M., \& Hommer, D. (2003). A region of mesial prefrontal cortex tracks monetarily rewarding outcomes: Characterization with rapid event-related fMRI. Neurolmage, 18(2), 263-272. http://dx.doi.org/10.1016 /S1053-8119(02)00057-5

Llinás, R., Ribary, U., Contreras, D., \& Pedroarena, C. (1998). The neuronal basis for consciousness. Philosophical Transactions of the Royal Society of London, Series B, Biological Sciences, 353(1377), 1841-1849. http://dx.doi.org /10.1098/rstb.1998.0336

Rubik, B. (2011). Neurofeedback-enhanced gamma brainwaves from the prefrontal cortical region of meditators and nonmeditators and associated subjective experiences. Journal of Alternative and Complementary Medicine, 17(2), 109-115. http://dx.doi.org/10.1089/acm.2009.0191

Sokhadze, E. (2012). Peak performance training 7 sing prefrontal EEG biofeedback. Biofeedback, 40(1), 7-15. http://dx.doi.org /10.5298/1081-5937-39.3.4

Sokhadze, E., \& Daniels, R. (2016). Effects of prefrontal $40 \mathrm{~Hz}-$ centered EEG band neurofeedback on emotional state and cognitive functions in adolescents. Adolescent Psychiatry, 6(4), 116-129.
Sokhadze, E., Stewart, C., El-Baz, A., Ramaswamy, R., Hollifield, M., \& Tasman, A. (2009). Induced EEG gamma oscillations in response to drug- and stress-related cues in cocaine addicts and patients with dual diagnosis. Journal of Neurotherapy, 13(4), 270-271.

Wang, Y., Sokhadze, E. M., El-Baz, A. S., Li, X., Sears, L., Casanova, M. F., \& Tasman, A. (2016). Relative power of specific EEG bands and their ratios during neurofeedback training in children with autism spectrum disorder. Frontiers in Human Neuroscience, 9, 723. http://dx.doi.org/10.3389 /fnhum.2015.00723

\section{Gender Differences in Quantitative EEG} Volumetric Analysis Shortly After Sport Concussion Injury in High School Athletes Harry Kerasidis, P. David Ims, Stacie Rector Chesapeake Neurology Associates, Prince Frederick, Maryland, USA

Introduction. We have previously reported changes in SLORETA quantitative analysis shortly after acute sport-related concussion injury in high school athletes, which persist after clinical recovery (Kerasidis \& Ims, 2017). We have also reported the effects of neurofeedback on these changes during the acute recovery period (Ims \& Kerasidis, 2018). Studies have identified gender differences in the incidence, severity, and recovery time from sport concussion injury, all increased in females (Cantu, 2010; Hamson-Utley et al., 2013; Miyashita, Diakogeorgiou, \& VanderVegt, 2016; Mollayeva, ElKhechen-Richandi, \& Colantonio, 2018; Tanveer, Zecavati, Delasobera, \& Oyegbile, 2017). The objective of this investigation is to explore gender differences in volumetric qEEG analysis after sport concussion injury in high school athletes.

Methods. Standard electroencephalograms (EEGs) were analyzed in 40 high school athletes (20 males) shortly after concussion injury using sLORETA imaging compared to a normative database (NYU/BrainDx). Peak Z-score variation (PZV), and percentage of volume of grey matter activity that fell outside $Z=-2.5$ to 2.5 (PIGMV for increased activity, PRGMV for reduced) were calculated for each of five EEG frequency bands.

Results. PZV was increased in the Delta/Theta/Alpha in both genders with no statistical gender difference (M/F averages: 3.82/3.16, $2.73 / 2.72,2.52 / 2.72$, respectively, $p>.05$ ); Beta in females, not males, Beta-Gamma in males and females which was significantly increased in females (M/F averages: 1.75/2.88, 3.64/5.02 respectively, $p<$ .01). PZV was decreased in Beta in males, not females (M/F averages: $-2.83 /-2.18, p<.05)$. There was a significant difference in reduced Beta-Gamma 
activity (M/F averages: $-1.11 /-0.49, p=.01)$. Greater than $1 \%$ grey matter volume of PIGMV was seen in Delta/Theta/Alpha/Beta and Beta-Gamma activity with no gender difference (M/F averages: 20.94/11.71, 5.87/7.38, 5.62/7.93, 4.09/9.22, p > .05). There was a significant difference in PIGMV in BetaGamma (M/F averages: 31.94/60.04, $p=.01$ ). Greater than 1\% PRGMV in Alpha/Beta in both genders and Theta activity in females, not males.

Conclusions. Slower frequency (Delta, Theta, and Alpha) abnormal variations show no statistical gender differences. In the faster frequency bands (Beta and Beta-Gamma), females demonstrate a larger variation from the norm and larger percent grey matter volume affected by increased Beta and BetaGamma activity. Males, not females, exhibit a deficiency in Beta activity after concussion. Further research to correlate these electrophysiologic changes with symptom severity and recovery time is needed.

\section{References}

Cantu, R. C. (2010). The role of concussion history and gender in recovery from soccer-related concussion. Yearbook of Sports Medicine, 2010, 29-30. http://dx.doi.org/10.1016/s01620908(10)79666-5

Hamson-Utley, J. J., Schulte, S., Fowler, L., Glodowski, C., Scharmann, S., Podlog, L., ... Ashley, A. (2013, July). Concussion-related neuroproteins: A comparison of gender differences in extreme sports. Poster presented at the 122nd Annual Conference of the American Psychological Association, Honolulu, HI. http://dx.doi.org/10.1037 le620352013-001

Ims, P. D., \& Kerasidis, H. (2018, April). Re-training the injured brain: A case series in sLORETA neurofeedback as an acute concussion intervention in youth. Poster session presented at the 49th Annual Association for Applied Psychophysiology and Biofeedback Conference, Orlando, FL.

Kerasidis, H., \& Ims, P. D. (2017, July). sLORETA quantitative EEG analysis demonstrates persistent EEG changes beyond clinical recovery from sport concussion in high school athletes: $A$ volumetric study. Poster session presented at 4th Annual American Academy of Neurology Sports Concussion Conference, Jacksonville, FL.

Miyashita, T. L., Diakogeorgiou, E., \& VanderVegt, C. (2016). Gender differences in concussion reporting among high school athletes. Sports Health, 8(4), 359-363. http://dx.doi.org/10.1177/1941738116651856

Mollayeva, T., El-Khechen-Richandi, G., \& Colantonio, A. (2018). Sex \& gender considerations in concussion research. Concussion, 3(1). http://dx.doi.org/10.2217/cnc-2017-0015

Tanveer, S., Zecavati, N., Delasobera, E. B., \& Oyegbile, T. O. (2017). Gender differences in concussion and postinjury cognitive rindings in an older and younger pediatric population. Pediatric Neurology, 70, 44-49. http://dx.doi.org /10.1016/j.pediatrneurol.2017.02.001
Social, Spiritual, Psychological, and Physiological Predictors of Well-being of Military Veterans: A Pilot Study of a Viable, Holistic, and Predictive Model of Well-being Manuel Halter

United States Special Operations Command (USSOCOM), Tampa, Florida, USA

Military leaders are striving to identify and implement innovative and necessary solutions to enhance or optimize military members' well-being, with the ultimate goal of improving the short- and long-term well-being of warriors and their families. This study tested the viability of a holistic model of well-being that was developed as a screening instrument. A secondary goal is to mitigate the detrimental effects of the high operational tempo and the extreme pressures faced by active duty military veteran community. Therefore, to be adequate the model had to be predictive of well-being to serve as such a baselining and monitoring tool. This new model involves a holistic, systems approach, integrating four key life domains that were hypothesized to impact overall well-being: human, psychological, social, and spiritual performance. These domains are interconnected and work together via situational, dispositional, and intentional variables to produce well-being, or the lack thereof (Howell, Kern, \& Lyubomirsky, 2007). To test the viability and utility of this model, a stepwise multiple regression analysis was conducted on archival data of 117 military veterans. Based on the literature of, and the shared nomological network between, well-being (e.g., Howell et al., 2007), PsyCap (e.g., Avey, 2014; Lorenz, Beer, Pütz, \& Heinitz, 2016), social isolation or connectedness (e.g., Kent, Hawthorne, Kjaer, Manniche, \& Albert, 2015), spiritual intelligence (e.g., Faraji, \& Begzadeh, 2017), psychological and cognitive performance (e.g., Del Brutto et al., 2015; Lathan, Spira, Bleiberg, Vice, \& Tsao, 2013), as well as heart rate variability (HRV; e.g., Fatisson, Oswald, \& Lalonde, 2016) and quantitative electroencephalogram (qEEG) metrics (e.g., Thatcher, North, Biver, \& Zhou, 2017), it was hypothesized that human performance (Brain Function Index or BFI [qEEG] and SDNN [HRV]), psychological performance (DASS-21 composite score), social performance (Friendship Scale composite score), and spiritual performance (SISRI24 composite score) would significantly predict wellbeing (Psychological Capital or PsyCap composite score). This set of predictors is hypothesized to account for a significant proportion of the well-being or PsyCap variance (i.e., CPC-12 composite scores; PsyCap). Furthermore, each predictor is hypothesized to explain a unique and significant 
proportion of the PsyCap variance. The expected results would suggest that the positive core construct of PsyCap can be predicted using self-report measures addressing each domain, combined with functional measures (i.e., BFI and SDNN) and cognitive performance assessment outcome measures (i.e., Defense Automated Neuropsychological Assessment [DANA]). Moreover, such findings would support a viable model of well-being, which military leaders can use to baseline and monitor its members.

\section{References}

Avey, J. B. (2014). The left side of psychological capital: New evidence on the antecedents of PsyCap. Journal of Leadership \& Organizational Studies, 21(2), 141-149. http://dx.doi.org/10.1177/1548051813515516

Del Brutto, O. H., Mera, R. M., Del Brutto, V. J., Maestre, G. E., Gardener, H., Zambrano, M., \& Wright, C. B. (2015). Influence of depression, anxiety and stress on cognitive performance in community-dwelling older adults living in rural Ecuador: Results of the Atahualpa Project. Geriatrics \& Gerontology International, 15(4), 508-514. http://dx.doi.org /10.1111/ggi.12305

Faraji, M., \& Begzadeh, S. (2017). The relationship between organizational commitment and spiritual intelligence with job performance in physical education staff in east Azerbaijan province. International Journal of Management, Accounting \& Economics, 4(5), 565-577.

Fatisson, J., Oswald, V., \& Lalonde, F. (2016). Influence diagram of physiological and environmental factors affecting heart rate variability: An extended literature overview. Heart International, 11(1), e32-e40. http://dx.doi.org/10.5301/heartint.5000232

Howell, R. T., Kern, M. L., \& Lyubomirsky, S. (2007). Health benefits: Meta-analytically determining the impact of well-being on objective health outcomes. Health Psychology Review, 1(1), 83-136. http://dx.doi.org/10.1080/17437190701492486

Kent, P., Hawthorne, G., Kjaer, P., Manniche, C., \& Albert, H. (2015). A Danish version of the Friendship Scale: Translation and validation of a brief measure of social isolation. Social Indicators Research, 120(1), 181-195. http://dx.doi.org /10.1007/s11205-014-0576-z

King, D. B., \& DeCicco, T. L. (2009). A viable model and self-report measure of spiritual intelligence. The International Journal of Transpersonal Studies, 28(1), 68-85.

Lathan, C., Spira, J. L., Bleiberg, J., Vice, J., \& Tsao, J. W. (2013). Defense Automated Neurobehavioral Assessment (DANA)Psychometric properties of a new field-deployable neurocognitive assessment tool. Military Medicine, 178(4), 365-371. http://dx.doi.org/10.7205/MILMED-D-12-00438

Lorenz, T., Beer, C., Pütz, J., \& Heinitz, K. (2016). Measuring psychological capital: Construction and validation of the Compound PsyCap Scale (CPC-12). PloS ONE, 11(4), 1-17. http://dx.doi.org/10.1371/journal.pone.0152892

Luthans, F., Youssef, C. M., Sweetman, D. S., \& Harms, P. D. (2013). Meeting the Leadership Challenge of Employee WellBeing Through Relationship PsyCap and Health PsyCap. Journal of Leadership \& Organizational Studies, 20(1), 118133. http://dx.doi.org/10.1177/1548051812465893

Thatcher, R. W., North, D. M., Biver, C. J. \& Zhou, L. (2017). Brain Function Index. Retrieved from

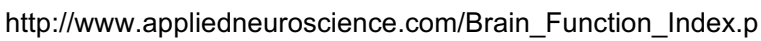
$\mathrm{df}$
Altered States NeuroMeditation: Current Approaches, Preliminary Findings, and Future Applications

Jeff Tarrant

NeuroMeditation Institute LLC, Corvallis, Oregon, USA

Using neurofeedback and other technologies to achieve altered states of consciousness has its roots in the early development of the field of neurofeedback. In fact, some of the initial neurofeedback protocols were designed to replicate many of the effects commonly experienced during a meditative state (Crane, 2007; Trudeau, 2016). Having observed that the practice of meditation often led to an increase in alpha power or increased theta activity crossing over alpha, these two approaches became the foundation of Deep States NeuroMeditation protocols, essentially attempting to facilitate elements of an altered state (Tarrant, 2017).

This work and these protocols are powerful and have been associated with impressive results with difficult clinical populations including alcoholics and those suffering with PTSD. Recent explorations into the study of consciousness has led to some new approaches and protocols for assisting clients into achieving altered states for the purposes of psychological and emotional healing.

This presentation will present a new and novel approach to achieving altered states with neurofeedback by replicating brain-based research on psychedelic therapies. Current research with psilocybin, LSD, DMT, and other psychedelics have all shown tremendous potential in treating a wide range of mental health disorders. It is believed that these impacts are due, at least in part, to the way they alter perception and the dysfunctional creation of a self-identity (Carhart-Harris et al., 2012). Based on this emerging field, certain brain patterns and brain regions have revealed themselves as important in these transformative experiences. By targeting these brain patterns through neurofeedback, in conjunction with additional strategies, we may be on the cusp of a brand-new approach to the use of neurofeedback. In this presentation, we will share preliminary research showing how these altered states can be facilitated using neurofeedback. We will explore approaches, indications and contraindications as well as a variety of adjunctive aids, including vibroacoustics, evocative music, and visual entrainment. 


\section{References}

Carhart-Harris, R. L., Erritzoe, D., Williams, T., Stone, J. M., Reed, L. J., Colasanti, A., ... Nutt, D. J., (2012). Neural correlates of the psychedelic state as determined by fMRI studies with psilocybin. Proceedings of the National Academy of Sciences (PNAS), 109(6), 2138-2143. http://dx.doi.org /10.1073/pnas.1119598109

Crane, R. (2007). Infinite potential: A neurofeedback pioneer looks back and ahead. In T. H. M. Press (Ed.), Handbook of neurofeedback: Dynamics and clinical applications: Haworth series in neurotherapy (pp. 3-21). Binghamton, NY: CYC Press.

Tarrant, J. (2017). Neuromeditation: An introduction and overview. In T. F. Collura \& J. A. Frederick (Eds.), Clinician's companion to QEEG and neurofeedback (annotated and with an introduction by J. Kiffer). New York, NY: Taylor \& Francis.

Trudeau, D. L. (2016). Experiences with alpha theta: Its origins in studies of meditation. In A. Martins-Mourao, \& C. Kerson (Eds.), Alpha-theta training in the 21st century: A handbook for clinicians and researchers (pp. 36-64). Murfreesboro, TN: Foundation for Neurofeedback and Neuromodulation Research (FNNR).

\section{Cognitive and Psychophysiological Test Operations as Assessment Tool for} Neurofeedback Clinicians: A Pilot Study on Its Preliminary Normative Data and Validity

Thomas Feiner ${ }^{1}$, Maria Juan ${ }^{2}$, Ruben Perez ${ }^{2}$

${ }^{1}$ Institute for EEG-Neurofeedback, Bavaria, Germany

${ }^{2}$ NEPSA Rehabilitación Neurológica, Salamanca, Spain

Neurofeedback is a paradigm in which individuals are trained to modulate their electroencephalogram (EEG) by providing them feedback about the targeted EEG component to treat symptoms and disorders associated to the neuronal condition. Neurofeedback shows effects on both the alleviation of symptoms and also changes in cognitive performance as an appreciated side effect or as bringing up the desired/expected ability like improved continuous attention, focus, and impulse control. For example, training the alpha band frequency has been associated with improved attentional control and working memory. Investigating attentional control is typically done with tasks such as the Stroop task, in which a color word is shown in a font color that is different (incongruent) than what the word represents and the participant is asked to name only the font color. Tests have shown that there is often a remarkable decrease in response time (delay) and an increase of accuracy from pre- to postneurofeedback sessions. In general, the Stroop test could be used to mark objectively success of neurofeedback over time. The problem is that that clinicians complain about the time which is spent to administer the Stroop test, which makes it interesting for research but not for clinical praxis.
The purpose of this study was to obtain normative data of a battery of informatized tests from the software Cognitive and Psychophysiological Test Operations (CAPITO) and its comparation with classical neuropsychological tests in order to assure construct validity with the goal to create a test which is suitable for use by primary care medical staff, psychologists, and neuropsychologists, since it can be administered in just 10 minutes.

We administered a battery of informatized tests (Stroop, simple reaction time, sustained attention, shifting attention) to 120 subjects who are cognitively normal and range in age from 18 to 65 years, of whom 26 randomly selected subjects also scored in classical tests in order to check battery validity (confidence level of $90 \%$, sampling margin of error $15 \%)$. In the case of subjects receiving both modalities (informatized and classical testing) the order of application was balanced, in order to avoid application order bias. Statistics of each test scores were calculated and comparisons between informatized and classical tests were conducted.

Normative data were collected for CAPITO battery and positive correlations with classical tests were found. No effects were found for age and sex in either test. Educational level impacted the Stroop test variables but not the other tests.

\section{References}

Brauer-Boone, K., Pontón, M. O., Gorsuch, R. L., González, J. J., \& Miller, B. L. (1998). Factor analysis of tour measures of prefrontal lobe functioning. Archives of Clinical Neuropsychology, 13, 585-595.

De la Torre, G. G. (2002). El modelo funcional de atención en neuropsicología. Revista de Psicología General y Aplicada, 55(1), 113-121.

Fan, J., McCandliss, B. D., Fossella, J., Flombaum, J. I., \& Posner, M. I. (2005). The activation of attentional networks. Neurolmage, 26(2), 471-479. http://dx.doi.org/10.1016 /j.neuroimage.2005.02.004

Fan, J., McCandliss, B. D., Sommer, T., Raz, M., \& Posner, M. I. (2002). Testing the efficiency and independence of attentional networks. Journal of Cognitive Neuroscience, 14(3), 340-347. http://dx.doi.org/10.1162/089892902317361886

Pineda, D. A., Merchán, V., Rosselli, M., \& Ardila, A. (2000). Estructura factorial de la función ejecutiva en estudiantes universitarios jóvenes $=$ Factor structure of the executive function in young university students. Revista de Neurología, 31(12), 1112-1118.

Posner, M. I., \& Dehaene, S. (1994). Attentional networks. Trends in Neurosciences, 17(2), 75-79.

Spikman, J., Kiers, H. A. L., Deelman, B. G., \& van Zomeren, A. H. (2001). Construct validity of concepts of attention in healthy controls and patients with $\mathrm{CHI}$. Brain and Cognition, 47(3), 446-460. http://dx.doi.org/10.1006/brcg.2001.1320 
Training Blood Flow: nHEG Utilization for

Specific qEEG Phenotypes in ASD

Adrian Van Deusen ${ }^{1,2}$, David Cantor ${ }^{3}$

${ }^{1}$ ITALLIS Communication Inc., Salvador, Brazil

${ }^{2}$ BrainDx LLC., Suwanee, Georgia, USA

${ }^{3}$ Mind and Motion Developmental Centers, Suwanee, Georgia, USA

The spectrum of autistic disorders is among the most heterogeneous, both in regard to electrophysiology and to metabolism. Specific to brain electrophysiology, research points to multiple noted outlying quantitative EEG features; consistent with the heterogeneity of the symptoms of this disorder. That variation of noted phenomenon has led to various EEG neurofeedback training strategies over the past two decades. Many of these strategies have undergone clinical research and, while encouraging, have presented similarly heterogeneous outcomes. It is still an active discussion with regard to which symptom features of ASD are specific to which neurofeedback protocols. Furthermore, protocols that address frontal pole delta or frontotemporal and temporal beta in low-functioning patients are challenging as these features are often confounded by muscle artifact and eye movement during training. In this presentation, we propose an alternative neurofeedback training protocol that can be used to improve particularly the frontal and temporal region dysfunctions that play a role in regulating attention and response control, and that is nearly impervious to the contamination of eye movement or muscle tension. One of the systemic dysregularities noted in the ASD population is a variety of metabolic imbalances which we argue can be presented as diffuse, low absolute power measures spanning two or more frequency bands in the qEEG. The specific physiological mechanism for this phenotypic pattern is not yet well understood but one rationale is that as a result of underlying metabolic dysregulation, oxygen perfusion is reduced resulting in reduced cell energy and subsequent low power. With this supposition, the authors have been using a nHEG (Toomim) training protocol since 2011. We have selected from 50 of the qualifying clinical nHEG training cases run in 2016 and 2017. The multicase study reveals significant improvement in the qEEG features from baseline to subsequent evaluations following approximately 10 hours of treatment. These results are compared to a protocol of neurofeedback therapy that trains to increase absolute power by rewarding EEG absolute power parameters alone.

\section{References}

Chabot, R. J., Coben, R., Hirshberg, L. \& Cantor, D. S. (2015). QEEG and VARETA based Neurophysiological Indices of Brain Dysfunction in Attention Deficit and Autistic Spectrum Disorder. Austin Journal of Autism \& Related Disabilities, 1(2), 1007.

Dias, A. M., Van Deusen, A. M., Oda, E., \& Bonfim, M. R. (2012) Clinical efficacy of a new automated hemoencephalographic neurofeedback protocol. Spanish Journal of Psychology, 15(3), 930-941.

Edelson, S. B., \& Cantor, D. S. (1998). Autism: Xenobiotic influence. Toxicology and Industrial Health, 14(6), 799-811. http://dx.doi.org/10.1177\%2F074823379801400603

Kouijzer, M. E. J., van Schie, H. T., Gerrits, B. J. L., Buitelaar, J. K., \& de Moor, J. M. H. (2013). Is EEG-biofeedback an Effective Treatment in Autism Spectrum Disorders? A Randomized Controlled Trial. Applied Psychophysiology and Biofeedback, 38(1), 17-28. http://dx.doi.org/10.1007/s10484012-9204-3

Wilcox, J., Tsuang, M. T., Ledger, E., Algeo, J., \& Schnurr, T. (2002). Brain perfusion in autism varies with age. Neuropsychobiology. 46, 13-16. http://dx.doi.org/10.1159 1000063570

\section{Applied Innovation in Clinical Practice — Let's Go Beyond Neurofeed back}

Amy Serin

Serin Center, Peoria, Arizona, USA

Many neurofeedback practitioners utilize multiple modalities in practice to enhance the effects of neurofeedback. Adjunct therapies such as counseling, transcranial direct current stimulation (tCDS), heart rate variability training, and biofeedback, among others, to improve patient outcomes. Two methodologies, bilateral alternating stimulation in tactile form (BLAST) and cranial electrical stimulation (CES) also show promise in altering electrical activity in key networks associated with stress (Feusner et al., 2012; Serin, Hageman, \& Kade, 2018) and can be used in conjunction with traditional neurofeedback. However, many clinicians do not have a model for how to apply these modalities in practice, nor have they reviewed the emerging data on the modalities. Beta EEG rhythm has been found to correlate to high situational and personal anxiety (Pavlenko, Chernyi, \& Goubkina, 2009) and BLAST has been found to significantly reduce beta activity, subjective distress and physiological body sensations in response to thinking about a stressful event (Serin et al., 2018) by possibly depotentiating amygdala activity (Harper, Rasolkhani-Kalhorn, \& Drozd, 2009) which is responsible activating the body's stress response (Ehrlich et al., 2009). The use of CES may result in cortical deactivation, may alter brain activity in the default mode network (DMN), and may create significant changes in intrinsic connectivity networks (Feusner et al., 2013). The body of literature is 
growing with regard to these two methodologies, and clinicians can utilize them in conjunction with traditional neurofeedback to achieve specific outcomes in treatment with patients with anxiety, insomnia, depression, and varied diagnoses. A review of clinical data, biometric data, EEG findings, and other research will be presented, along with guidelines for clinical use of the modalities and a system and structure for incorporation into clinical practice. Discussion of how to combine these modalities will also be summarized to advance the field of applied neurofeedback.

\section{References}

Busscher, B., Spinhoven, P., van Gerwen, L. J., \& de Geus, E. J. C. (2013). Anxiety sensitivity moderates the relationship of changes in physiological arousal with flight anxiety during in vivo exposure therapy. Behaviour Research and Therapy, 51(2), 98-105. http://dx.doi.org/10.1016/j.brat.2012.10.009

Ehrlich, I., Humeau, Y., Grenier, F., Ciocchi, S., Herry, C., \& Lüthi, A. (2009). Amygdala inhibitory circuits and the control of fear memory. Neuron, 62(6), 757-771. http://dx.doi.org/10.1016 /j.neuron.2009.05.026

Feusner, J. D., Madsen, S., Moody, T. D., Bohon, C., Hembacher, E., Bookheimer, S. Y., \& Bystritsky, A. (2012). Effects of cranial electrotherapy stimulation on resting state brain activity. Brain and Behavior, 2(3), 211-220. http://dx.doi.org /10.1002/brb3.45

Harper, M. L., Rasolkhani-Kalhorn, T., \& Drozd, J. F. (2009). On the neural basis of EMDR therapy: Insights from qEEG studies. Traumatology, 15(2), 81-95. http://dx.doi.org/10.1177 /1534765609338498

Lande, R. G., \& Gragnani, C. (2013). Efficacy of cranial electric stimulation for the treatment of insomnia: A randomized pilot study. Complementary Therapies in Medicine, 21(1), 8-13. http://dx.doi.org/10.1016/j.ctim.2012.11.007

Pavlenko, V. B., Chernyi, S. V., \& Goubkina, D. G. (2009). EEG correlates of anxiety and emotional stability in adult healthy subjects. Neurophysiology, 41(5), 337-345. http://dx.doi.org /10.1007/s11062-010-9111-2

Serin, A., Hageman, N. S., \& Kade, E. (2018). The therapeutic effect of bilateral alternating stimulation tactile form technology on the stress response. Journal of Biotechnology and Biomedical Science, 1(2), 42-47. http://dx.doi.org /10.14302/issn.2576-6694.jbbs-18-1887

\section{Multivariate Coherence Training for \\ Developmental Trauma}

Robert Coben ${ }^{1}$, Clark Thompson ${ }^{2}$, Anne Stevens ${ }^{1}$

${ }^{1}$ Integrated Neuroscience Services, Fayetteville, Arkansas, USA

${ }^{2}$ BaySide NeuroRehab Services, Portland, Maine, USA

Developmental trauma is a major public health concern that has generated increased interest from researchers over the past few decades. The Adverse Childhood Experiences (ACE) study revealed correlational relationships between traumatic childhood experiences and an array of outcomes after several years, which included depression, substance abuse, domestic violence, suicide attempts, and various medical conditions (Felitti et al., 1998). Common domains of impairment observed by children exposed to developmental trauma are multifaceted, consisting of self-concept, attachment, behavioral regulation, affect regulation, dissociation, and biology (Cook et al., 2017). Neuroimaging studies of this population have revealed structural changes in the brain, such as reduced development of the hippocampus, amygdala, corpus callosum, and left neocortex (Teicher et al., 2003). Overall, there are a paucity of neurofeedback studies on developmental trauma. A small handful of projects have focused on power training (van der Kolk et al., 2016), some of which have been qEEG based (Huang-Storms, Bodenhamer-Davis, Davis, \& Dunn, 2006).

We are conducting a study on participants with a history of developmental trauma who underwent neurofeedback training. We hypothesize that subjects who undergo neurofeedback training will show significantly decreased levels of mood and trauma-related symptoms compared to controls. Based on the findings of Armes and Coben (2017), we hypothesize that changes in connectivity will be related to success in neurofeedback and reduction of symptoms. Our study consists of 40 participants who were randomly assigned to a four-channel multivariate coherence training group or a control group who received an alternative treatment with no neurofeedback training. Dependent variables included the Beck Depression Inventory-II, Beck Anxiety Inventory, Trauma Symptom Inventory-II, as well as power and graph theory connectivity metrics based on qEEG findings. These measures were all administered at time 1 and time 2 with an intervening period of neurofeedback training. Preliminary findings show enhancements in coherence metrics are associated with decreased depression, anxiety, and trauma-related symptoms.

\section{References}

Armes, C. A., \& Coben, R. (2017, September). Impact of developmental trauma on brain function and connectivity. Presented at the International Society of Neurofeedback and Research 25th Annual Conference, Foxwoods, CT.

Cook, A., Spinazzola, J., Ford, J., Lanktree, C., Blaustein, M., Cloitre, M., ... van der Kolk, B. (2005). Complex trauma in children and adolescents. Psychiatric Annals, 35(5), 390-398.

Felitti, V. J., Anda, R. F., Nordenberg, D., Williamson, D. F., Spitz, A. M., Edwards, V., ... Marks, J. S. (1998). Relationship of childhood abuse and household dysfunction to many of the leading causes of death in adults: The Adverse Childhood Experiences (ACE) Study. American Journal of Preventive Medicine, 14(4), 245-258. http://dx.doi.org/10.1016/S07493797(98)00017-8

Huang-Storms, L., Bodenhamer-Davis, E., Davis, R., \& Dunn, J. (2006). QEEG-guided neurofeedback for children with histories 
of abuse and neglect: Neurodevelopmental rationale and pilot study. Journal of Neurotherapy, 10(4), 3-16. http://dx.doi.org/10.1300/J184v10n04_02

Teicher, M. H., Andersen, S. L., Polcari, A., Anderson, C. M., Navalta, C. P., \& Kim, D. M. (2003). The neurobiological consequences of early stress and childhood maltreatment. Neuroscience \& Biobehavioral Reviews, 27(1), 33-44. http://dx.doi.org/10.1016/S0149-7634(03)00007-1

van der Kolk, B. A., Hodgdon, H., Gapen, M., Musicaro, R., Suvak, M. K., Hamlin, E., \& Spinazzola, J. (2016). A randomized controlled study of neurofeedback for chronic PTSD. PLOS ONE 11(12), e0166752. http://dx.doi.org /10.1371/journal.pone.0166752

\section{The Effect of Infraslow Frequency \\ Neurofeedback on Quantitative \\ Electroencephalogram and Autonomic \\ Nervous System Function in Adults with \\ Anxiety and Related Diseases \\ Karlien Balt ${ }^{1}$, Mark Smith ${ }^{2}$, Peet Du Toit ${ }^{1}$, Priyesh Bipath $^{1}$ \\ ${ }^{1}$ University of Pretoria, Pretoria, Gauteng, South Africa \\ ${ }^{2}$ Neurofeedback Services of New York, New York, New York, USA}

Background. Over the last decade it has been observed in clinical practice that Infraslow Frequency (ISF) training shifts clients in physiological state during training. Peripheral body temperature, pupil size, and breathing rate are a few examples of autonomic nervous system (ANS) responses regularly observed during ISF neurofeedback training. ISF electroencephalographic (EEG) biofeedback focuses on the low energy signals produced by the brain. This includes frequencies of less than $0.1 \mathrm{~Hz}$ (Smith, Collura, Ferrera, \& de Vries, 2014). Evidence suggests that these slow oscillations play a role in synchronizing faster activity and modulates cortical excitability (Bazhenov \& Timofeev, 2006). The origins of these slow oscillations are not yet well understood but studies have indicated the involvement of the thalamus and other subcortical structures (Lörincz, Geall, Bao, Crunelli, \& Hughes, 2009). The ANS is an important role player in maintaining sympatheticparasympathetic and cardiovascular homeostasis. It includes vagal cholinergic and sympathetic noradrenergic nerves that supply the heart and sympathetic noradrenergic nerves that enmesh arterioles. Therefore, clinicians and researchers have long sought valid, noninvasive, quantitative means to identify patho-physiologically relevant abnormalities of these systems (Goldstein, Bentho, Park, \& Sharabi, 2011). Heart Rate Variability (HRV) is one of the most well-known means of measurement. There is increasing research pointing to the clinical application of HRV in training and exercise due to its apparent result in strengthening sympathetic-parasympathetic balance (Peper, Harvey, Lin, Tylova, \& Moss, 2007). Achieving an increased HRV while doing ISF training should be a good indicator of firstly reaching clients Optimum Frequency (OF) and secondly achieving a sympathetic-parasympathetic balance (Camp, Remus, Kalburgi, Porterfield, \& Johnson, 2012; Collura, 2014). This study hypothesizes that ISF training has a measurable physiological effect on an individual by measuring certain autonomic functions; namely, HRV, muscle tension, skin temperature, skin conductance, heart rate, respiration rate, and blood pressure. Also, to demonstrate how ISF training impacts the resting state EEG.

Methods. Thirty adults between the ages of 18 and 55 with primarily anxiety will receive a quantitative electroencephalogram (qEEG) to get a baseline before training. The participants will then receive ISF neurofeedback training for 10 sessions while continuous monitoring of ANS changes will be done to determine if there are measurable changes. After 10 sessions we will repeat a qEEG to determine what changes occurred. The same process will be completed for a control group. The control group will receive one-channel power training where Theta and Hibeta activity will be inhibited at 3-7 Hz and 22-30 $\mathrm{Hz}$ respectively and Lobeta $12-15 \mathrm{~Hz}$ activity enhanced at the $\mathrm{C} 4$ location on the head.

Anticipated results. Preliminary results and a pilot study conducted show significant changes that have been observed in participants trained in ISF neurofeedback, both in the activation patterns when looking at the GEEG and the autonomic functions that were measured. No significant changes have been seen thus far in the control group.

Conclusion. The study will possibly demonstrate that autonomic functions are affected by ISF neurofeedback training and that changes occur in the resting state EEG of participants trained.

\section{References}

Bazhenov, M., \& Timofeev, I. (2006). Thalamocortical oscilations. Scholarpedia, 1(6), 1319. http://dx.doi.org/10.4249 /scholarpedia.1319

Camp, R. M., Remus, J. L., Kalburgi, S. N., Porterfield, V. M., \& Johnson, J. D. (2012). Fear conditioning can contribute to behavioral changes observed in a repeated stress model. Behavioural Brain Research, 233(2), 536-544. http://dx.doi.org/10.1016/j.bbr.2012.05.040

Collura, T. F. (2014). Technical foundations of neurofeedback. New York, NY: Routlege/Taylor \& Francis

Goldstein, D. S., Bentho, O., Park, M. Y., \& Sharabi, Y. (2011). Low-frequency power of heart rate variability is not a measure of cardiac sympathetic tone but may be a measure of 
modulation of cardiac autonomic outflows by baroreflexes. Experimental Physiology, 96(12), 1255-1261. http://dx.doi.org /10.1113/expphysiol.2010.056259

Hallman, D., \& Lyskov, E. (2012). Autonomic regulation in musculoskeletal pain. Retrieved on July 15, 2017, from Intech Open Science: https://www.intechopen.com/books/pain-inperspective/autonomic-regulation-in-musculosceletal-pain

Lőrincz, M., Geall, F., Bao, Y., Crunelli, V., \& Hughes, S. W. (2009). ATP-dependent infra-slow $(<0.1 \mathrm{~Hz})$ oscillations in thalamic networks. PLoS One, 4(2), e4447. http://dx.doi.org /10.1371/journal.pone.0004447

Peper E, Harvey, R., Lin, I., Tylova, H., \& Moss, D. (2007). Is there more to blood volume pulse than heart rate variability, respiratory sinus arrhythmia, and cardiorespiratory synchrony? Biofeedback, 35(2), 54-61.

Smith, M. L., Collura, T. F., Ferrera, J., \& de Vries, J. (2014). Infraslow fluctuation training in clinical practice: A technical history. NeuroRegulation, 1(2), 187-207. http://dx.doi.org /10.15540/nr.1.2.187

\section{The Human Compassion Circuit \\ Larry Stevens}

Northern Arizona University, Flagstaff, Arizona, USA

Compassion has been one of the most cherished, acclaimed, practiced, and pursued of human virtues for literally thousands of years - and a foremost part of nearly all organized religions and spiritual quests. Despite its lofty and celebrated status, tragically there are far too many examples in human history of "the far enemy" of Compassion, Cruelty. For a species so enamored with this cherished human virtue, how are we so able to engage in cruel acts? Perhaps the answer, in part, to this critical social question lies in our neurological makeup; perhaps there are specialized structures in our brains that are hardwired for the experience of Compassion, and structures that are similarly hardwired for our expression of Cruelty.

This presentation reports on a recent literature review, analysis, and integration by the author of a growing body of compassion research, detailed in his chapter entitled, "The Brain that Longs to Care for Others: The Current Neuroscience of Compassion" in his soon-to-be-released academic textbook The Neuroscience of Empathy, Compassion, and SelfCompassion (Elsevier/ Academic Press, June 2018). This review suggests that such hardwired circuits do indeed exist in each of our brains. A follow-up research investigation by the author was designed to begin the answer to the question above and to more clearly and with greater certainty identify these circuits.

This presentation will thus clarify the temporal and spatial characteristics of a neurological circuit in the human brain identified with the experience of Compassion. The hypothesis of the reported research investigation is that participants will respond to careworthy and to blameworthy compassion scenarios with a specified differential, sequential (temporal) spatial circuit involving (1) an affective amygdala (AMG), anterior insular cortex (AIC), and anterior cingulate cortex (ACC) sub-circuit; (2) motor intentional mirroring structures in the Mirror Neuron System of the premotor cortex (PMC) and inferior parietal lobe (IPL); (3) cognitive regulatory subcircuits in the dorsolateral prefrontal cortex (dIPFC), ventrolateral PFC (vIPFC), dorsomedial PFC (dmPFC), and posterior temporal cortex (PTC); and then (4) simultaneous with the PFC subcircuits above, self-other differentiation Theory of Mind (ToM) subcircuits in the bilateral temporal parietal junction (TPJ), precuneus (PCun), and dmPFC. These temporal and spatial pathways are identified by continuous electroencephalograph (EEG) recordings and during subsequent power spectral and LORETA neuroimaging analyses following the participant's experiencing of specific, visuallypresented compassion scenarios. Exciting Neurotherapy, TMS, Meditation, and Psychotherapy protocols are explored as ways of increasing Compassion in human beings, perhaps even as alternatives to one of the cruelest of compassion "far enemies," torture.

\section{References}

Buhle, J. T., Silvers, J. A., Wager, T. D., Lopez, R., Onyemekwu, C., Kober, H., ... Ochsner, K. N. (2014). Cognitive reappraisal of emotion: A meta-analysis of human neuroimaging studies. Cerebral Cortex, 24(11), 2981-2990. http://dx.doi.org/10.1093 /cercor/bht154

de Waal, F. (2009). The age of empathy. New York, NY: Three Rivers Press.

Fehse, K., Silveira, S., Elvers, K., \& Blautzik, J. (2015). Compassion, guilt and innocence: An fMRI study of responses to victims who are responsible for their fate. Social Neuroscience, 10(3), 243-252. http://dx.doi.org/10.1080 /17470919.2014.980587

Gallese, V., Keysers, C., \& Rizzolatti, G. (2004). A unifying view of the basis of social cognition. Trends in Cognitive Sciences, 8(9), 396-403. http://dx.doi.org/10.1016/j.tics.2004.07.002

Goetz, J. L., Keltner, D., \& Simon-Thomas, E. (2010). Compassion: An evolutionary analysis and empirical review. Psychological Bulletin, 136(3), 351-374. http://dx.doi.org/ 10.1037/a0018807

Kédia, G., Berthoz, S., Wessa, M., Hilton, D., \& Martinot, J.-L. (2008). An agent harms a victim: A functional magnetic resonance imaging study on specific moral emotions. Journal of Cognitive Neuroscience, 20(10), 1788-1798. http://dx.doi.org/10.1162/jocn.2008.20070

Lindquist, K. A., Wager, T. D., Kober, H., Bliss-Moreau, E., \& Barrett, L. F. (2012). The brain basis of emotion: A metaanalytic review. Behavioral and Brain Sciences, 35(3), 121 143. http://dx.doi.org/10.1017/S0140525X11000446

Lutz, A., Greischar, L. L., Rawlings, N. B., Ricard, M., \& Davidson, R. J. (2004). Long-term meditators self-induce high-amplitude gamma synchrony during mental practice. Proceedings of the National Academy of Sciences, 101(46), 16369-16373. http://dx.doi.org/10.1073/pnas.0407401101 
Ochsner, K. N., Ray, R. D., Cooper, J. C., Robertson, E. R., Chopra, S. Gabrieli, J. D. E, \& Gross, J. J. (2004). For better or for worse: Neural systems supporting the cognitive down- and up-regulation of negative emotion. Neurolmage, 23(2), 483499. http://dx.doi.org/10.1016/j.neuroimage.2004.06.030

Ochsner, K. N., Ray, R. R., Hughes, B., McRae, K., Cooper, J. C., Weber, J., ... Gross, J. J. (2009). Bottom-up and top-down processes in emotion generation: Common and distinct neural mechanisms. Psychological Science, 20(11), 1322-1331. http://dx.doi.org/10.1111/j.1467-9280.2009.02459.x

Phan, K. L., Wager, T., Taylor, S. F., \& Liberzon, I. (2002). Functional neuroanatomy of emotion: A meta-analysis of emotion activation studies in PET and fMRI. Neurolmage, 16(2), 331-348. http://dx.doi.org/10.1006/nimg.2002.1087

Porges, S. W. (2003). The Polyvagal Theory: Phylogenetic contributions to social behavior. Physiology and Behavior, 79, 503-513. http://dx.doi.org/10.1016/S0031-9384(03)00156-2

Premack, D., \& Woodruff, G. (1978). Does the chimpanzee have a theory of mind? Behavioral and Brain Sciences, 1(4), 515-526. http://dx.doi.org/10.1017/S0140525X00076512

Singer, T. (2006). The neuronal basis and ontogeny of empathy and mind reading: Review of literature and implications for future research. Neuroscience and Behavioral Reviews, 30(6), 855-863. http://dx.doi.org/10.1016/j.neubiorev.2006.06.011

Singer, T., Critchley, H. D., \& Preuschoff, K. (2009). A common role of insula in feelings, empathy and uncertainty. Trends in Cognitive Sciences, 13(8), 334-340. http://dx.doi.org/10.1016 /j.tics.2009.05.001

\section{Neurofeedback: An Effective Treatment for Symptoms of Posttraumatic Stress Disorder in Veterans \\ Connie McReynolds \\ California State University, San Bernardino, California, USA}

This presentation was published previously in this journal as:

McReynolds, C. J., Bell, J., \& Lincourt, T. M. (2017). Neurofeedback: A noninvasive treatment for symptoms of posttraumatic stress disorder in veterans. NeuroRegulation, 4(3-4), 114-124. http://dx.doi.org/10.15540/nr.4.3-4.114

To read the full article go to: http://www.neuroregulation.org/article/view/17962/11 657

\section{POSTERS PRESENTATIONS}

\section{The Frontal Alpha Asymmetry and}

Neurofeedback in Patients with Major

Depressive Disorder

I-Mei Lin ${ }^{1}$, Ya-Ting Hung ${ }^{1}$, Sheng-Yu Fan ${ }^{2}, Y u L^{3}{ }^{3}$, Nien-Mu Chiu ${ }^{3}$, Chi-Fa Hung ${ }^{3}$, Cheng-Fang Yen ${ }^{1}$, YiChun Yeh', Mei-Feng Huang ${ }^{1}$, Tai Ling Liu' ${ }^{1}$, Peng-Wei Wang ${ }^{1}$, Huang Chi Lin ${ }^{1}$

${ }^{1}$ Kaohsiung Medical University, Kaohsiung City, Taiwan, Taiwan ${ }^{2}$ National Cheng Kung University, Taiwan, Taiwan

${ }^{3}$ Kaohsiung Chang Gung Memorial Hospital, Kaohsiung City, Taiwan, Taiwan

Background and Description. Previous study indicated that Frontal Alpha Asymmetry (FAA) is a biomarker for patients with Major Depressive Disorder (MDD; Davidson, 1998). Asymmetry scores (A1) were calculated from log-transformed of alpha power (8-12 Hz), log (F4) - log (F3). Some studies based on theoretical of FAA and applied alpha asymmetry neurofeedback (ALAY) showed some improvement in the depressive symptoms. However, the changes of electroencephalography (EEG) parameters were inconsistent (Baehr, Rosenfeld, \& Baehr, 2001; Cheon, Koo, \& Choi, 2016; Choi et al., 2011; Wang et al., 2016). This study hypothesized the differences on EEG patterns between participants with FAA (A1-) and without FAA (A1+) among the healthy controls and patients with MDD; as well as the effect of ALAY neurofeedback on A1 score between the $A 1(-)$ group or the $A 1(+)$ group among patients with MDD.

Method. Study 1: The participants were composed of 127 patients with MDD (72 in the A1+ group; 55 in the A1- group) and 129 healthy controls (87 in the A1+ group; 42 in the A1-group). Beck Depression Inventory-II was administered, and a 19-channel EEG cap with BrainAvatar (BrainMaster, Bedford, Ohio) has collected EEG raw signals at F3 and F4 and transformed to absolute alpha power $(8-12 \mathrm{~Hz})$, and then calculated alpha asymmetry score (A1). Study 2: A total of 48 patients with MDD were assigned to the ALAY neurofeedback groups $(\mathrm{A} 1+, n=11$; $\mathrm{A} 1-$, $n=13)$ and the control groups (A1+, $n=10 ; \mathrm{A} 1-, n=$ 12) based on their $A 1$ score at pretest. The ALAY neurofeedback groups received $60 \mathrm{~min}$, twice a week for 10 consecutive sessions of neurofeedback that was assisted by BioGraphy Infiniti 6.0 (Thought Technology, Quebec, Canada). The goal of neurofeedback was to increase A1 score with the visual and the auditory feedback. The control group received the normal treatment as usual. All of the participants underwent pretest and posttest measurement which included the performance on 
psychological questionnaires and EEG, which included the F3 alpha, F4 alpha, and A1 score.

Results. For EEG patterns, there was higher alpha power in the left prefrontal lobe $(F 3)$ in the $A 1(-)$ group compared to $A 1(+)$ group in the healthy controls; as well as lower alpha power in the right prefrontal lobe (F4) in the $A 1(-)$ group compared to A1 (+) group in patients with MDD. Regarding the effect of ALAY neurofeedback, the ALAY A1(-) group significantly increased the $A 1$ score at posttest than the pretest. However, ALAY A1(+) group did not show significant improvements, as well as two MDD controls (A1+ and $A 1-)$. Both neurofeedback groups (ALAY A1- and ALAY A1+) significantly decreased the depression total score and cognitive depression, and ALAY A1(-) also decreased the anxiety score.

Conclusion. The high alpha power in the left prefrontal lobe in the healthy controls, and low alpha power in the right prefrontal lobe in the MDD group, were found in participants who had FAA. The neurofeedback therefore was beneficial for patients with FAA in decreasing depressive symptoms and increasing the A1 score.

\section{References}

Baehr, E., Rosenfeld, J. P., \& Baehr, R. (2001). Clinical use of an alpha asymmetry neurofeedback protocol in the treatment of mood disorders: Follow-up study one to five years post therapy. Journal of Neurotherapy, 4(4), 11-18. http://dx.doi.org/10.1300/J184v04n04_03

Cheon, E.-J., Koo, B.-H., \& Choi, J.-H. (2016). The efficacy of neurofeedback in patients with major depressive disorder: An open labeled prospective study. Applied Psychophysiology and Biofeedback, 41(1), 103-110. http://dx.doi.org/10.1007 /s10484-015-9315-8

Choi, S. W., Chi, S. E., Chung, S. Y., Kim, J. W., Ahn, C. Y., \& Kim, H. T. (2011). Is alpha wave neurofeedback effective with randomized clinical trials in depression? A pilot study. Neuropsychobiology, 63(1), 43-51. http://dx.doi.org/10.1159 1000322290

Davidson, R. J. (1998). Anterior electrophysiological asymmetries, emotion, and depression: Conceptual and methodological conundrums. Psychophysiology, 35(5), 607-614.

Wang, S.-Y., Lin, I.-M., Peper, E., Chen, Y.-T., Tang, T.-C., Yeh, Y.-C., ... Chu, C.-C. (2016). The efficacy of neurofeedback among patients with major depressive disorder: Preliminary study. NeuroRegulation, 3(3), 127-134. http://dx.doi.org /10.15540/nr.3.3.127
A Real-time fMRI Neurofeedback for Mild to Severe Depression Compared to Frontal Alphaasymmetry Neurofeedback and CognitiveBehavioral Therapy

Mikhail Melnikov ${ }^{1}$, Mark Shtark ${ }^{1}$, Dmitriy Bezmaternykh ${ }^{1}$, Lyudmila Kozlova ${ }^{1}$, Mikhail Pokrovski ${ }^{2}$, Andrey Savelov ${ }^{2}$, Estate Sokhadze ${ }^{3}$, Kira Natarova ${ }^{4}$, Evgeniy Petrovskiy $^{2}$, Tatiana Syrtchina ${ }^{1}$, Tatiana Andamova ${ }^{1}$

${ }^{1}$ Federal Research Center of Fundamental and Translational Medicine, Novosibirsk, Novosibirskaya Oblast', Russia

${ }^{2}$ International Tomography Center SB RAS, Novosibirsk, Novosibirskaya Oblast', Russia

${ }^{3}$ University of South Carolina, School of Medicine Greenville, Greenville, South Carolina, USA

${ }^{4}$ International Institute of Psychology and Psychotherapy, Novosibirsk, Novosibirskaya Oblast', Russia

Background. For decades EEG neurofeedback has been the only method of self-regulation of brain activity in mental disorders. Current developments in fMRI technology made possible neuroimaging neurofeedback targeted to a well-defined cerebral area. Implementations of the technology aimed to enhance subjects' control of the activity of brain structures involved in emotion regulation were successful both in healthy volunteers (Johnston et al., 2011) and in patients with major depression (Hamilton et al., 2016; Linden et al., 2012; Young et al., 2017).

Objectives. The aim of our study was to examine effects of the real-time fMRI neurofeedback as a treatment arm for mild to severe depression. Alphaasymmetry neurofeedback and cognitive-behavioral therapy (CBT) served as control treatment arms.

Methods. Thirty subjects (10 males, 20 females, aged 20-50, mean age of 33) were recruited and randomly assigned to experimental or one of two control groups. Participants of experimental group received eight weekly sessions of fMRI neurofeedback targeted bilaterally to the area within medial prefrontal cortex. During each session blocks of enhancing and suppressing the response of the target area were alternating. Subjects continuously received visual feedback reflecting percent of signal change within the region of interest (ROI). The source signal was recorded using Philips Ingenia 3T MR scanner with an EPI sequence, TR $=1000 \mathrm{~ms}$. Temporal dynamics of the signal from ROI was captured from the IViewBOLD graphs and presented on the screen as a yellow circle, the diameter and brightness of which depended on the signal values. Offline fMRI analysis was performed using SPM 12 software. Concurrent EEG was recorded with a 32channel MR-compatible BrainAmp system, corrected for MR, cardiac, and ocular artifacts and processed in 
EEGLab software. First control group patients received sixteen 25-min sessions of frontal alphaasymmetry neurofeedback. Participants from the second control group were treated with eight individual and eight group sessions of CBT. Each subject underwent psychiatric examination (MADRS), psychological assessment (BDI, SDS [Zung SelfRating Depression Scale], HADS), and EEG-fMRI recording at rest and during performing an emotionally salient task at start, at middle, and at the end of the course.

Results. Patients from all the groups significantly improved from the treatment. A status of some patients according to DSM- 5 changed to milder depression or to no depression condition. The fMRIneurofeedback group showed significant improvements on MADRS, BDI, SDS, and HADS that were statistically comparable with those in alphaasymmetry neurofeedback and CBT. Patients of the fMRI group demonstrated ability to control prefrontal cortex signal both in usual feedback and in transfer (no feedback) sessions and gained positive changes of emotional state during sessions.

Conclusions. FMRI-based neurofeedback holds a promise for a targeted regulation of emotional circuits and can be considered as a potentially clinically efficacious technique of self-regulation in mood disorders. However, cost-benefit ratio remains a problem for this application. Studies of EEG-fMRI correlates during the real-time fMRI-neurofeedback sessions may be instrumental for enhancing EEG neurofeedback treatment protocols.

\section{References}

Hamilton, J. P., Glover, G. H., Bagarinao, E., Chang, C., Mackey, S., Sacchet, M. D., \& Gotlib, I. H. (2016). Effects of saliencenetwork-node neurofeedback training on affective biases in major depressive disorder. Psychiatry Research, 249, 91-96. http://dx.doi.org/10.1016/j.pscychresns.2016.01.016

Johnston, S., Linden, D. E. J., Healy, D., Goebel, R., Habes, I., \& Boehm, S. G. (2011). Upregulation of emotion areas through neurofeedback with a focus on positive mood. Cognitive Affective, \& Behavioral Neuroscience, 11(1), 44-51. http://dx.doi.org/10.3758/s13415-010-0010-1

Linden, D. E. J., Habes, I., Johnston, S. J., Linden, S., Tatineni, R., Subramanian, L., ... Goebel, R. (2012). Real-time selfregulation of emotion networks in patients with depression. PLoS One, 7(6), 38115. http://dx.doi.org/10.1371 /journal.pone.0038115

Young, K. D., Siegle, G. J., Zotev, V., Phillips, R., Misaki, M., Yuan, H., ... Bodurka, J. (2017). Randomized clinical trial of real-time fMRI amygdala neurofeedback for major depressive disorder: Effects on symptoms and autobiographical memory recall. The American Journal of Psychiatry, 174(8), 748-755. http://dx.doi.org/10.1176 /appi.ajp.2017.16060637
Preliminary Evidence for Stress-Reducing Effects of Bilateral Alternating Stimulation Tactile (BLAST) Following Significant Quantitative Electroencephalography (qEEG) Reduction in Beta Wave Activity

Amy Serin ${ }^{1}$, Dominic Di Loreto ${ }^{1}$, Emily Kade ${ }^{2}$

${ }^{1}$ Serin Center, Peoria, Arizona, USA

${ }^{2}$ Midwestern University, Phoenix, Arizona, USA

Bilateral alternating stimulation in tactile form (BLAST) technology has been found to significantly reduce subjective distress and physiological body sensations in response to thinking about a stressful event (Serin, Hageman \& Kade, 2018). TouchPoints are noninvasive devices that deliver BLAST and are believed to reduce sympathetic nervous system arousal associated with anxiety (Busscher, Spinhoven, van Gerwen, \& de Geus, 2013) by depotentiating amygdala activity (Harper, Rasolkhani-Kalhorn, \& Drozd, 2009) responsible for activating the body's stress response (Ehrlich et al., 2009). Beta EEG rhythm has been found to correlate to high situational and personal anxiety (Pavlenko, Chernyi, \& Goubkina, 2009). The purpose of this study was to utilize quantitative electroencephalography (qEEG) recordings to identify significant changes in electrical brain activity upon thinking of a stressful event and subsequently upon the delivery of BLAST. It was hypothesized that upon thinking of a stressful event beta activity would increase and subsequently reduce significantly upon delivery of BLAST.

Methods. A total of 21 participants (9 male, 12 female), ages 7 to 63 ( $M$ age $=27.8 ; S D=16.5$ ), participated in the study and were recruited through the Serin Center. The sample consisted of 14 clinical participants with heterogeneous diagnoses of anxiety, major depressive disorder, and attentiondeficit/hyperactivity disorder. The remaining sample consisted of nonclinical participants. QEEG data was collected at the Serin Center's locations in Peoria and Scottsdale, Arizona. Data was collected utilizing a NeuroField Q20 amplifier and was stored using NeuroGuide by Applied Neuroscience Inc. Participants underwent a 5-min baseline recording followed by an instruction to think about a stressful event. Participants were then asked to hold TouchPoints devices. 19-channel qEEG recordings were taken while thinking about the stressful event, during the delivery of BLAST (holding the TouchPoints), and then a baseline was taken again upon removal of the TouchPoints. Paired $t$-test analysis was conducted before and after BLAST with NeuroGuide's Neurostat software. 
Results. Preliminary EEG recordings comparing the stress condition to the TouchPoints condition exhibited significantly reduced activity in frontal Theta, specifically in $5 \mathrm{~Hz}$ at Fp2 and F4 sites and reduced activity in Beta 1 at $12-14 \mathrm{~Hz}$ in the frontal channel locations (Fp1, Fp2, Fp3, Fz, F4). Significant right frontal decreases are shown in Beta 2 at 16-18 $\mathrm{Hz}$, Beta 3 bands at $19 \mathrm{~Hz}$ and $23 \mathrm{~Hz}$, and Gamma 1 at $30-35 \mathrm{~Hz}$ with activity decreasing along the midline also in Beta 3 at $19 \mathrm{~Hz}$ and $23 \mathrm{~Hz}$ and in Gamma 1 at $30 \mathrm{~Hz}$.

Conclusion. The significant reduction in beta activity provides preliminary evidence that BLAST technology may have a therapeutic effect on reducing subcortical activity associated with anxiety and stress. Our results are consistent with previous studies (Pavlenko et al., 2009) suggesting beta wave activity is correlated with increased levels of anxiety. This preliminary data implicates the potential efficacy of BLAST as a mediator of SNS arousal and stress through beta-activity reduction in both clinical and nonclinical samples. Follow-up research is required utilizing a comparison control group with a larger sample to assess for qEEG differences in brain activity.

\section{References}

Busscher, B., Spinhoven, P., van Gerwen, L. J., \& de Geus, E. J. C. (2013). Anxiety sensitivity moderates the relationship of changes in physiological arousal with flight anxiety during in vivo exposure therapy. Behaviour Research and Therapy, 51(2), 98-105. http://dx.doi.org/10.1016/j.brat.2012.10.009

Ehrlich, I., Humeau, Y., Grenier, F., Ciocchi, S., Herry, C., \& Lüthi, A. (2009). Amygdala inhibitory circuits and the control of fear memory. Neuron, 62(6), 757-771. http://dx.doi.org/10.1016 /j.neuron.2009.05.026

Harper, M. L., Rasolkhani-Kalhorn, T., \& Drozd, J. F. (2009). On the neural basis of EMDR therapy: Insights from qEEG studies. Traumatology, 15(2), 81-95. http://dx.doi.org/10.1177 $/ 1534765609338498$

Pavlenko, V. B., Chernyi, S. V., \& Goubkina, D. G. (2009). EEG correlates of anxiety and emotional stability in adult healthy subjects. Neurophysiology, 41(5), 337-345. http://dx.doi.org /10.1007/s11062-010-9111-2

Serin, A., Hageman, N. S., \& Kade, E. (2018). The therapeutic effect of bilateral alternating stimulation tactile form technology on the stress response. Journal of Biotechnology and Biomedical Science, 1(2), 42-47. http://dx.doi.org /10.14302/issn.2576-6694.jbbs-18-1887
Event-related Potential Study of Illusory Figure Processing Deficits in Children with Autism

Spectrum Disorder

Estate Sokhadze'; Ayman El-Baz'; Desmond Kelly3; Ricardo Jose De Leon'; Thomas Feiner'; Manuel Casanova $^{1}$

${ }^{1}$ University of South Carolina School of Medicine Greenville, Greenville, South Carolina, USA

'University of Louisville, Louisville, Kentucky, USA ${ }^{3}$ Greenville Health System, Greenville, South Carolina, USA ${ }^{4}$ University of Santiago de Compostela, San Pedro Sula, Honduras ${ }^{5}$ Institute for EEG-Neurofeedback, Bavaria, Germany

Background. Analysis of event-related potentials (ERP) is one of the most effective methods of investigation of information-processing stages in the brain. ERP methodology represents a valuable technique to study normative cognitive processes in typically developing (TD) subjects and, at the same time, may serve as a sensitive tool to assess differences in individuals with autism spectrum disorder (ASD). It has been shown in visual and auditory modalities in various types of oddball tasks that children with ASD present abnormalities in ERPs (Bomba \& Pang, 2004; Kemner, van der Gaag, Verbaten, \& van Engeland, 1999). Both the frontal P3a to novel stimuli and the parietal P3b to attended target stimuli were reported to be abnormal in autism (Cui, Wang, Liu, \& Zhang, 2017; Townsend et al., 2001).

Objectives. In a series of studies (Baruth, Casanova, Sears, \& Sokhadze, 2010; Sokhadze, Baruth, El-Baz, et al., 2010; Sokhadze, Baruth, Tasman, et al., 2009) using various oddball tasks we showed that group differences between ASD and TD children can be found for both attended and nonattended stimuli not only in late potentials (P3a, P3b) but also in early ERPs (P100, N100) and response-locked ERP (ERN). Among oddball tasks the three-stimulus visual oddball with illusory figures was most informative for this purpose. The goal of the study was investigation of group differences in ERP recorded at midline frontal and parietal sites for determination if these topographies are reflecting atypicality of ERP in the ASD.

Methods. Seventy children with ASD and 30 typical children performed on an oddball task with illusory figures. EEG was collected using a 128-channel EEG system. The task involved the recognition of a specific illusory shape, in this case a square or triangle, created by three or four inducer disks (Kanizsa, 1976). The regions-of-interest (ROI) for ERP analysis were only frontal and parietal midline areas. 
Results. Children with ASD did not differ from typical children in reaction time (RT), but they committed more errors $(12.9 \%$ vs. $2.2 \%, F=14.9, p<.001)$ and did not show normative posterror RT slowing $(F=$ 27.6, $p<.001)$. The error-related negativity was lower in ASD $(F=8.5, p=.004)$. The early ERPs (P100 and N100) to nontarget stimuli were of higher amplitude and delayed in the ASD group ( $p s<0.05$ ). The late ERPs (P3a and P3b) to nontarget stimuli were prolonged in ASD without amplitude differences, though P3a was delayed as well to targets in the ASD (458 vs. $426 \mathrm{~ms}, F=4.9, p=.03$ ).

Conclusions. Results are in concordance with our prior studies where children with ASD showed excessive reactivity to task-irrelevant stimuli at the early sensory stage processing of information leading to delayed cognitive ERP to targets resulting in error monitoring and correction deficits. It was important to replicate these findings when ERPs were analyzed only at the midline frontal and parietal areas as it may have practical implications. It creates opportunity for our group to start development of a custom-made experimental control and EEG acquisition system with limited number of channels (e.g., Fz, Pz) for ERP analysis in oddball test with illusory figures that can be used for functional diagnostic and as outcome of neurofeedback or neuromodulation interventions.

\section{References}

Baruth, J. M., Casanova, M., Sears, L., \& Sokhadze, E. (2010). Early-stage visual processing abnormalities in high-functioning autism spectrum disorder (ASD). Translational Neuroscience, 1(2), 177-187. http://dx.doi.org/10.2478/v10134-010-0024-9

Bomba, M. D., \& Pang, E. W. (2004). Cortical auditory evoked potentials in autism: A review. International Journal of Psychophysiology, 53(3), 161-169. http://dx.doi.org/10.1016 /j.ijpsycho.2004.04.001

Cui, T., Wang, P. P., Liu, S., \& Zhang, X. (2017). P300 amplitude and latency in autism spectrum disorder: A meta-analysis. European Child \& Adolescent Psychiatry, 26(2),177-190. http://dx.doi.org/10.1007/s00787-016-0880-z

Kanizsa, G. (1976). Subjective contours. Scientific American, 234(4), 48-52.

Kemner, C., van der Gaag, R. J., Verbaten, M., \& van Engeland, H. (1999). ERP differences among subtypes of pervasive developmental disorders. Biological Psychiatry, 46(6), 781789. http://dx.doi.org/10.1016/S0006-3223(99)00003-7

Sokhadze, E., Baruth, J., El-Baz, A., Horrell, T., Sokhadze, G., Carroll, T., ... Casanova, M. F. (2010). Impaired error monitoring and correction function in autism. Journal of Neurotherapy, 14(2), 79-95. http://dx.doi.org/10.1080 /10874201003771561

Sokhadze, E. M., Baruth, J. M., Sears, L., Sokhadze, G. E., El-Baz, A. S., Williams, E. L., ... Casanova, M. F. (2012). Event-related potential study of attention regulation during illusory figure categorization task in ADHD, autism spectrum disorder, and typical children. Journal of Neurotherapy, 16(1), 12-31. http://dx.doi.org/10.1080/10874208.2012.650119

Sokhadze, E., Baruth, J., Tasman, A., Sears, L., Mathai, G., ElBaz, A. \& Casanova, M. F. (2009). Event-related potential study of novelty processing abnormalities in autism. Applied Psychophysiology and Biofeedback, 34(1), 37-51. https://doi.org/10.1007/s10484-009-9074-5

Townsend, J., Westerfield, M., Leaver, E., Makeig, S., Jung, T.-P., Pierce, K., \& Courchesne, E. (2001). Event-related brain response abnormalities in autism: Evidence for impaired cerebello-frontal spatial attention networks. Cognitive Brain Research, 11(1), 127-145. http://dx.doi.org/10.1016/S09266410(00)00072-0

\section{Effects rTMS-based Neuromodulation Dosage} on Event-related Potentials and Evoked and Induced Gamma Oscillations in Children with Autism Spectrum Disorder

Estate Sokhadze'; Eva Lamina'; Emily Casanova ${ }^{1}$; Desmond Kelly2; Ayman El-Baz'; Guela Sokhadze ${ }^{3}$; Manuel Casanova ${ }^{1}$

${ }^{1}$ University of South Carolina School of Medicine Greenville, Greenville, South Carolina, USA

${ }^{2}$ Greenville Health System, Greenville, South Carolina, USA

${ }^{3}$ University of Louisville, Louisville, Kentucky, USA

Background. Autism is defined as a spectrum of behavioral disorders that have in common impairments in social interaction and communication skills, language deficits, and a restricted repertoire of interests and stereotyped activities. There are several theoretical models of the neuropathology of autism spectrum disorders (ASD), and one of them suggests the presence of an excessive cortical excitation/inhibition (E/I) ratio (Casanova, Buxhoeveden, \& Gomez, 2003; Rubinstein \& Merzernich, 2003; Uzunova, Pallanti, \& Hollander, 2016) that affects functional connectivity. This model explains atypical event-related potential (ERP) and evoked and induced gamma oscillations observed in ASD during task performance. Repetitive transcranial magnetic stimulation (rTMS), especially using low-frequency inhibitory stimulation, can be considered as a method of modulating the E/I bias.

Objectives. In our prior exploratory studies (Sokhadze, Baruth, et al., 2010; Sokhadze, El-Baz, et al., 2009) we used different schedules of rTMS to investigate outcomes of rTMS in ASD. In this study, 124 high functioning ASD children $(I Q>80$, less than 18 years of age) were recruited and assigned to either a waitlist group or one of three different number of weekly rTMS sessions (i.e., $6,12,18$ ) to investigate effects of dosage on functional and behavioral outcomes. The project was aimed at selection of more effective length of rTMS course.

Methods. TMS consisted of trains of $1.0 \mathrm{~Hz}$ pulses applied over dorsolateral prefrontal cortex. The experimental task was a three-stimulus visual oddball with illusory Kanizsa figures. Behavioral response 
variables included reaction time and error rate along with EEG indices such as ERP and evoked and induced gamma oscillations. One hundred and twelve patients completed the assigned number of rTMS sessions.

Results. We found significant positive changes from baseline to post-TMS treatment period in motor responses accuracy (lower percentage of committed errors, restored normative posterror slowing), in ERP indices and in evoked and induced gamma responses. Parental reports showed significant reductions in aberrant behavior scores as well as decreased scores of repetitive and stereotypic behaviors. The gains of outcomes increased with the total number of treatment sessions. Results of our clinical research study showed most significant changes from baseline in functional measures of performance in oddball task and in behavioral symptom ratings following 18 sessions of rTMS treatment. Several measures showed a difference from baseline and waitlist in reaction time and ERP/EEG variables after 12 sessions of rTMS, but only a few of them reached statistical significance after a six-session rTMS course.

Conclusions. Our results suggest that rTMS, particularly after 18 sessions, facilitates cognitive control, attention and target stimuli recognition by improving discrimination between task-relevant and task-irrelevant illusory figures in an oddball test. Improvement in executive functions and behavioral symptoms of autism further suggests that TMS has the potential to target core features of ASD. The results of this dosage-response study could serve as important prerequisites that could inform the planning of a blinded randomized clinical trial. Among potential implications of the study should be considered potential of combining rTMS with neurofeedback training (Sokhadze et al., 2014) aimed at reinforcement of neuromodulation effects using operant conditioning in similar manner as reported by our group earlier.

\section{References}

Casanova, M. F., Buxhoeveden, D., \& Gomez, J. (2003). Disruption in the inhibitory architecture of the cell minicolumn: Implications for autism. The Neuroscientist, 9(6), 496-507. http://dx.doi.org/10.1177/1073858403253552

Rubenstein, J. L. R., \& Merzenich, M. M. (2003). Model of autism: Increased ratio of excitation/inhibition in key neural systems. Genes, Brain and Behavior, 2(5), 255-267. http://dx.doi.org /10.1034/j.1601-183X.2003.00037.x

Sokhadze, E., Baruth, J., Tasman, A., Mansoor, M., Ramaswamy, R., Sears, L., ... Casanova, M. F. (2010). Low-frequency repetitive transcranial magnetic stimulation (rTMS) affects event-related potential measures of novelty processing in autism. Applied Psychophysiology and Biofeedback, 35(2) 147-161. http://dx.doi.org/10.1007/s10484-009-9121-2

Sokhadze, E. M., El-Baz, A., Baruth, J., Mathai, G., Sears, L., \& Casanova, M. F. (2009). Effects of low-frequency repetitive transcranial magnetic stimulation (rTMS) on gamma frequency oscillations and event-related potentials during processing of illusory figures in autism. Journal of Autism and Developmental Disorders, 39(4), 619-634. http://dx.doi.org /10.1007/s10803008-0662-7

Sokhadze, E. M., El-Baz, A. S., Tasman, A., Sears, L. L., Wang, Y., Lamina, E. V., \& Casanova, M. F. (2014). Neuromodulation integrating rTMS and neurofeedback for the treatment of autism spectrum disorder: An exploratory study. Applied Psychophysiology and Biofeedback, 39(3-4), 237-257. http://dx.doi.org/10.1007/s10484-014-9264-7

Uzunova, G., Pallanti, S., \& Hollander, E. (2016). Excitatory/inhibitory imbalance in autism spectrum disorders: Implications for interventions and therapeutics. The World Journal Biological Psychiatry, 17(3), 174-186. http://dx.doi.org/10.3109/15622975.2015.1085597

\section{Using Neurofeedback to Lower Anxiety Symptoms: A Follow-up Study \\ Mark S. Jones, Heather Hitsman}

The University of Texas at San Antonio, San Antonio, Texas, USA

Introduction. Anxiety represents one of the most commonly diagnosed mental illnesses in the United States, affecting approximately $18 \%$ of the population annually (NIMH, 2017). This retrospective study intended to assess whether qEEG-guided amplitude neurofeedback (NF) is viable in symptom reduction of anxiety. This presentation updates a previously presented and published pilot study on treating anxiety symptoms with neurofeedback, based on data from 2014 to 2015 (Dreis et al., 2015). The pilot study involved a retrospective assessment of the efficacy of qEEG-guided one-channel neurofeedback for reduction of anxiety symptoms. The treatment was provided through an urban on-campus community counseling center operated by a university counseling department for the training of master and doctoral level students in neurofeedback. This updated retrospective study follows the same model as the pilot study with increased study size, based on data from 2014 to 2018.

Methods. From 2014 to 2018, 76 total clients were assessed and treated for anxiety using neurofeedback. Retrospectively, 34 clients met inclusion criteria for data analysis. Inclusion required that primary symptoms be related to anxiety, that the client is naive to neurofeedback and completion of pre- and postassessments including qEEG and symptom checklists. It is projected that an additional 12 clients may be included in the final analysis to be concluded by August 2018. Clients of the counseling center were from the local community. Demographics include age ranges from 11 to $61(M=$ 
34.76, $S D=15.15), 18$ male and 16 female; 17 identified as Caucasian, 13 identified as Hispanic/Latino, and 3 Caucasian/Hispanic ethnicity; one declined to identify their ethnicity. Pre- and postassessments included qEEG, the Zung SelfRating Anxiety Scale, Screen for Child AnxietyRelated Disorders (SCARED), and the Achenbach System of Empirically Based Assessment (ASEBA). Clients received between 20 to 30 minutes of qEEGguided NF treatment sessions, twice a week. The range of attended session was $4-19(M=12.14, S D$ $=3.24$ ).

Results. On the Zung Anxiety Scale mean scores were reduced from 45.5 to 38.1 with $t(32)=7.20, p<$ $.0001, \mathrm{~d}=1.22$. SCARED mean scores were reduced from 37.22 to 23.27 with $t(2)=4.80, p<.041$, $d=2.77$. ASEBA mean scores were reduced on the Anxious-Depressed scale from 68.61 to 64.19 with $t(35)=3.49, p<.001, d=0.58$, on the Anxiety Disorder (DSM) scale from 65.14 to 61.97 with $t(35)$ $=2.57, p<.014, d=0.43$, and on the Total Problems scale (average of eight core scales) from 59.53 to
55.19 with $t(35)=3.85, p<.0001, d=0.64$. Limitations and directions for future research will be discussed along with details of qEEG markers and related treatment protocols.

\section{References}

Dreis, S. M., Gouger, A. M., Perez, E. G., Russo, G. M., Fitzsimmons, M. A., \& Jones, M. S. (2015). Using neurofeedback to lower anxiety symptoms using individualized qEEG protocols: A pilot study. NeuroRegulation, 2(3), 137148. http://dx.doi.org/10.15540/nr.2.3.137

Lu, Y., Wang, C., Su, L., Ma, Z., Li, S., \& Fan, Y. (2017). Effects of neurofeedback on panic disorder patients' anxiety. NeuroQuantology, 15(3). http://dx.doi.org/10.14704 Inq.2017.15.3.1083

Mennella, R., Patron, E., \& Palomba, D. (2017). Frontal alpha asymmetry neurofeedback for the reduction of negative affect and anxiety. Behaviour Research and Therapy, 92, 32-40. http://dx.doi.org/10.1016/j.brat.2017.02.002

Received: November 11, 2018

Accepted: November 11, 2018

Published: December 7, 2018 